\title{
Activity-dependent LTP in the dentate gyrus promotes epileptic seizures
}

\author{
Kaoutsar Nasrallah ${ }^{1}$, M. Agustina Frechou ${ }^{1,4}$, Young J. Yoon ${ }^{1,3}$, Subrina Persaud ${ }^{1}$, \\ Tiago Gonçalves ${ }^{1,4}$ and Pablo E. Castillo ${ }^{1,2}$ *
}

${ }^{1}$ Dominick P. Purpura Department of Neuroscience, ${ }^{2}$ Department of Psychiatry \& Behavioral Sciences, ${ }^{3}$ Department of Anatomy and Structural Biology, ${ }^{4}$ Gottesman Institute for Stem Cell Biology and Regenerative Medicine, Albert Einstein College of Medicine, Bronx NY 10461, U.S.A.

Running title: LTP at hippocampal Mossy Cell-Granule Cell synapse promotes seizures

Keywords: Hippocampus, Mossy cell, epilepsy, granule cell, BDNF, TrkB receptor, presynaptic, LTP, epilepsy, optogenetic, DREADD

* To whom correspondence should be addressed:

Pablo E. Castillo, MD/PhD

Dominick P. Purpura Department of Neuroscience

Albert Einstein College of Medicine

1410 Pelham Parkway South

Kennedy Center, Room 703

Bronx, NY 10461, USA

Email: pablo.castillo@einsteinmed.org

Title: 9 words

Total number of words

Abstract: 150; Introduction: 440; Results: 2,564; Discussion: 1,413; Methods: 2,609

Figures: 5

Suppl figures: 3 
Epilepsy is a devastating brain disorder whose cellular mechanisms remain poorly understood. Excitatory mossy cells (MCs) in the dentate gyrus of the hippocampus are implicated in temporal lobe epilepsy, the most common form of focal epilepsy in adults. However, the role of MCs during initial seizures, before MC loss occurs, is unclear. Here, we show that initial seizures induced with kainic acid (KA) intraperitoneal injection in adult mice, a well-established model of experimental epilepsy, not only increased MC and granule cell (GC) activity in vivo, but also triggered a BDNFdependent long-term potentiation at MC-GC synapses (MC-GC LTP). In vivo induction of MC-GC LTP worsened KA-induced seizures, whereas selective MC silencing and Bdnf genetic removal from GCs, which abolishes LTP, were both anti-epileptic. Thus, initial seizures strengthen MCGC synaptic transmission, thereby promoting epileptic activity. Our findings reveal a potential mechanism of epileptogenesis that may help develop therapeutic strategies for early intervention. 


\section{INTRODUCTION}

Epilepsy is a common neurological disorder characterized by recurrent epileptic seizures, often associated with profound cognitive, psychological, and social deleterious consequences ${ }^{1}$. About $30 \%$ of the patients are resistant to antiepileptic drugs that do not necessarily target pathogenic mechanisms involved in epileptogenesis ${ }^{2}$. To develop more effective treatments, a better understanding of the cellular and molecular processes implicated in the early stages of epilepsy, before the brain damages become irreversible, is required. Mossy cells (MCs), excitatory neurons in the dentate gyrus (DG) of the hippocampus, play a critical role in temporal lobe epilepsy (TLE) ${ }^{3-}$ 5 , the most common form of focal epilepsy in adults ${ }^{6}$. However, whether MC activity can be proor anti-epileptic has been a subject of debate for several decades ${ }^{3-5,7-10}$. MC loss is a hallmark feature of chronic TLE in both humans and animal models ${ }^{11-15}$. Recent studies reported that while surviving MCs in a mouse model of chronic TLE play an antiepileptic role ${ }^{4}$, these cells could be pro-epileptic early during initial experimental seizures ${ }^{5}$.

Recurrent excitatory activity is a core mechanism in epilepsy ${ }^{16}$. In the DG, glutamatergic MCs and granule cells (GCs) are reciprocally connected thus forming an intrinsic excitatory loop. Remarkably, a single MC makes more than 30,000 synaptic contacts onto GCs, locally, contralaterally, and along the longitudinal axis of the hippocampus ${ }^{17,18}$. Furthermore, repetitive stimulation of MC axons in vitro, induces robust long-term potentiation at MC-GC excitatory synapses (MC-GC LTP). The DG is characterized by very sparse activation of GCs ${ }^{19-21}$ and it is believed to act as a gate that opens during epileptic seizures ${ }^{22,23}$. The long-lasting strengthening of MC-GC synaptic transmission is sufficient to overcome the basal strong GC inhibition, thereby allowing MCs to drive GCs and presumably open the DG gate ${ }^{24}$. MC-GC LTP is mediated by brain-derived neurotrophic factor (BDNF)/Tropomyosin receptor kinase B (TrkB) signaling ${ }^{24,25}$, which is known to promote TLE $^{26,27}$. Therefore, activity-dependent strengthening of MC-GC synapses may promote epilepsy through the extensive MC projections onto GCs. While an episode of prolonged seizures (e.g. status epilepticus) can result in TLE ${ }^{28-31}$, it is unknown whether and how initial seizures can impact MC-GC synaptic strength.

Using multiple complementary approaches, such as chemogenetics, in vitro electrophysiology, in vivo optogenetics, in vivo calcium imaging and a conditional knockout strategy, we found that initial seizures not only increased MC and GC activity, in vivo, but also triggered a BDNFdependent strengthening of MC-GC synaptic transmission. In addition, in vivo induction of MC- 
GC LTP was sufficient to promote convulsive seizures, whereas interfering with BDNF signaling and $\mathrm{MC}$ activity had an anti-convulsant effect. Our findings support a pro-epileptic role of MCs and BDNF in early TLE and provide a potential causal link between MC-GC LTP and epilepsy.

\section{RESULTS}

\section{Chemogenetic silencing of MCs reduced acute kainic acid-induced seizures}

To test the hypothesis that MC-GC LTP has a pro-convulsant effect during early epilepsy, we first tested the prediction that silencing MCs should reduce the severity/susceptibility of experimental seizures induced by a single intraperitoneal (IP) injection of kainic acid (KA, $30 \mathrm{mg} / \mathrm{kg}$ ), a wellestablished experimental model of epilepsy ${ }^{32,33}$. To suppress $M C$ output the $G_{i}(h M 4 D i)$ inhibitory designer receptor exclusively activated by designer drug (iDREADD) was selectively expressed in MCs. We bilaterally injected a Cre-recombinase-dependent virus expressing the iDREADD (AAV-CaMKII-DIO-hM4D $\left(G_{i}\right)$-mCherry) into the DG of Drd2-Cre mice, whereas Drd2-Cre mice injected with AAV-CaMKII-DIO-mCherry served as control (Fig 1A). Consistent with previous reports $^{5,34}$, we found that the viral expression was selective to MCs (Fig 1B). Next, we verified that the iDREADD efficiently responded to the DREADD agonist clozapine N-oxide (CNO). Bath application of $10 \mu \mathrm{M}$ CNO significantly reduced MC-GC EPSC amplitude in acute slices obtained from Drd2-Cre mice injected with AAV-CaMKII-DIO-hM4D $\left(\mathrm{G}_{\mathrm{i}}\right)$-mCherry but not with AAV-CaMKIIDIO-mCherry (Fig $1 \mathrm{C}$ and D, iDREADD: $43.8 \pm 4.3 \%$ of baseline, $n=6, p<0.001$, paired t-test; control: $100.6 \pm 5.5 \%$ of baseline, $n=5, p>0.05$, paired t-test). We then monitored and scored behavioral seizures induced by KA IP injection (30 mg/kg) for 2 hours (see Methods) in both Drd2Cre mice injected with AAV-CaMKII-DIO-hM4D(Gi)-mCherry (iDREADD) or AAV-CaMKII-DIOmCherry (control). The two groups were injected with CNO (2 mg/kg) 30 minutes prior KA IP administration (30 mg/kg). Consistent with a recent study using the pilocarpine-model ${ }^{5}$, we found that silencing MCs reduced seizure severity and susceptibility, as indicated by significant decrease in the total cumulative seizure score (Fig $1 \mathrm{~F}$, two way ANOVA RM, AAV condition: $F(1$, $5)=7.2, p<0.05$, time: $F(1.1,5.6)=71.7, p<0.001$; AAV condition $x$ time: $F(1,5)=88.1, p<$ 0.001 ), in sum score (Fig $1 \mathrm{G}$, control: $33.7 \pm 5.1, \mathrm{n}=7$; iDREADD: $17.8 \pm 3.2, \mathrm{n}=6$; control vs iDREADD: $p<0.05$, unpaired t-test) and increase in latency to stage 3 (Fig $1 \mathrm{H}$, control: $45.7 \pm$ 6.1, $n=7$; iDREADD: $91.7 \pm 14.9, \mathrm{n}=6$; control vs iDREADD: $\mathrm{p}<0.05$, unpaired t-test). These results reinforce the notion that $\mathrm{MC}$ activity has a pro-convulsant effect during initial epileptic seizures. 


\section{Initial convulsive seizures potentiated MC-GC transmission presynaptically}

We hypothesized that initial epileptic seizures may increase MC activity, and thus induce MC-GC LTP in vivo. MC repetitive activity is sufficient to trigger a robust MC-GC LTP in acute brain slices obtained from healthy rodents ${ }^{24}$. Furthermore, a recent in vivo study using calcium indicator and fiber photometry reported that DG neuronal activity is increased during KA-induced seizures ${ }^{35}$. However, the contribution of specific subtypes of neurons, including MCs and GCs, remains unknown. We therefore monitored $M C$ and $\mathrm{GC}$ activity in vivo using calcium imaging during acutely induced seizures. To this end, we expressed the genetically encoded $\mathrm{Ca}^{2+}$ indicator jRGECO1a selectively in DG excitatory neurons, by unilaterally injecting AAV-CaMKII-jRGECO1a into the DG of wild type (WT) adult mice. The animals were then implanted with a chronic imaging window above the dorsal hippocampus and MC and GC activity was visualized using head-fixed 2 photon imaging before and during acute seizures (Fig 2A, B). After collecting basal activity, seizures were induced with a single KA IP injection $(20 \mathrm{mg} / \mathrm{kg})$. Neuronal activity was monitored during stage 3 of convulsive seizures, which was determined by the presence of forelimb clonus. Saline-injected mice served as a control. In total, we recorded $94 \mathrm{MCs}$ and $136 \mathrm{GCs}$ from 4 salineinjected mice and $63 \mathrm{MCs}$ and $66 \mathrm{GCs}$ from $5 \mathrm{KA}$-injected mice. We found that both MC (Fig $2 \mathrm{C}$ and $D$, saline: $-0.06 \pm 0.07, N=4, p>0.05$, t-test; $K A: 7.6 \pm 1.4, N=5, p<0.01$, t-test) and $G C$ (Fig $2 \mathrm{C}$ and $\mathrm{D}$, saline: $-0.04 \pm 0.08, \mathrm{~N}=4, \mathrm{p}>0.05$, t-test; $\mathrm{KA}: 6.4 \pm 1.8, \mathrm{~N}=5, \mathrm{p}<0.05$, t-test) calcium signals were significantly increased during $K A$-induced convulsive seizures but not after saline administration, indicating a robust increase in neuronal activity.

We then tested whether initial epileptic seizures, by increasing MC activity and inducing LTP, could have strengthen MC-GC connections in vivo. We analyzed MC-GC synaptic transmission in both KA-injected and saline-injected mice. After a single KA injection ( $20 \mathrm{mg} / \mathrm{kg}$, IP), the animals were monitored and sacrificed for acute hippocampal slice preparation once stage 3 of convulsive seizures was reached (Fig 2E). Sham-injected mice were used as control. MC-GC synaptic function was assessed by activating MC axons while performing whole-cell voltage-clamp recordings from GCs. We found that MC-GC synaptic transmission was significantly strengthened, as indicated by an increase in the input/output function (Fig 2F, two way ANOVA $\mathrm{RM}$, IP injection: $F(1,9)=6.5, p<0.05$, stimulation intensity: $F(1.4,12.5)=64.5, p<0.001$; IP injection $x$ stimulation intensity: $F(1,9)=139.8, p<0.001$ ), while both paired-pulse ratio (PPR) and coefficient of variation $(\mathrm{CV})$ were significantly reduced in KA-injected as compared to saline- 
treated mice (Fig 2G, PPR: saline: $1.31 \pm 0.08, n=10 ; \mathrm{KA}: 1.01 \pm 0.06, \mathrm{n}=10$; saline vs KA: $\mathrm{p}<$ 0.05, unpaired t-test; CV: saline: $0.44 \pm 0.06, n=10 ; K A: 0.28 \pm 0.02, n=10$; saline vs KA: $p<$ 0.05 , unpaired t-test). These results strongly suggest that initial seizures, presumably by inducing presynaptic LTP, strengthened MC-GC synapses in vivo. If so, this plasticity should be occluded in hippocampal slices prepared from KA-injected mice. In support of this hypothesis, we found that both synaptically-induced LTP by repetitive stimulation of MC axons (Fig $2 \mathrm{H}$, saline: $155.5 \pm$ $14.7 \%$ of baseline, $n=6, p<0.05$, paired t-test; KA: $99.2 \pm 3.8 \%$ of baseline, $n=5, p>0.05$, paired t-test; saline vs KA: $p<0.05$, unpaired t-test) and chemically-induced LTP by transient application (10 $\mathrm{min}, 50 \mu \mathrm{M}$ ) of the adenylyl-cyclase activator forskolin ${ }^{24}$ (Fig 2I, saline: $170.7 \pm 7.6$ $\%$ of baseline, $n=9, p<0.001$, paired t-test; KA: $114.3 \pm 9.8 \%$ of baseline, $n=6, p>0.05$, paired t-test; saline vs KA: $p<0.001$, unpaired t-test), were impaired in KA-injected mice as compared to control (Fig $2 \mathrm{H}$ and I). Altogether, our findings strongly suggest that early $\mathrm{KA}$-induced seizures strengthened MC-GC synaptic transmission by inducing MC-GC LTP in vivo.

\section{Blocking seizure-induced MC-GC LTP had an anti-convulsant effect}

BDNF/TrkB signaling is critical for MC-GC LTP. BDNF is released, by both MCs and GCs, upon repetitive presynaptic activity and is necessary and sufficient for the induction of MC-GC LTP (Berthoux et al. $)^{24}$. To test whether BDNF is also involved in the seizure-induced strengthening of MC-GC synaptic transmission, occurring in vivo, we conditionally $\mathrm{KO}$ Bdnf from GC, a manipulation that abolishes MC-GC LTP (Berthoux et al.), and tested whether seizures can trigger MC-GC potentiation in absence of postsynaptic BDNF. Given that experimental seizures can be reduced in Bdnf KO mice ${ }^{36,37}$, we only injected $0.5 \mu \mathrm{l}$ of AAV5.CaMKII.Cre-mCherry or AAV5.CaMKII.mCherry (control) into the DG upper blade of $B d n f^{\text {fl/fl }}$ mice in order to prevent a potential failure in seizure induction when knocking out Bdnf from DG excitatory neurons. Mice were sacrificed $25 \mathrm{~min}$ after KA injection, which is the average time for reaching stage 3 convulsive seizure in WT control mice. We then prepared acute slice to monitor MC-GC synaptic function (Fig 3A). While behavioral seizures were comparable in control and cKO mice (all animals reached stage 3 of convulsive seizures by minute 25), Bdnf deletion from GCs (Cre-mCherry+ GCs) prevented seizure-induced MC-GC LTP, as KA IP injection failed to increase MC EPSC amplitude (Fig 3B, two way ANOVA RM, IP injection: $F(1,6)=0.003, p>0.05$, stimulation intensity: $F(1.56,9.38)=57.97, p<0.001$; IP injection $x$ stimulation intensity: $F(1,6)=287.4, p<$ 0.001 ) or decrease PPR (Fig 3C, saline: $1.2 \pm 0.01, n=7 ; \mathrm{KA}: 1.2 \pm 0.1, n=7$; saline vs $\mathrm{KA}: \mathrm{p}>$ 0.05 , unpaired t-test) and CV (Fig $3 C$, saline: $0.40 \pm 0.04, n=7 ; K A: 0.42 \pm 0.06, n=7$; saline vs 
$\mathrm{KA}: \mathrm{p}>0.05$, unpaired t-test) as compared to saline-injected mice. The lack of KA-induced synaptic strengthening was not due to viral expression since $K A$ injection efficiently increased the input/output function (MC EPSC amplitude, Fig 3D, two way ANOVA RM, IP injection: F $(1,4)=$ 23.6, $p<0.01$, stimulation intensity: $F(1.34,5.34)=68.9, p<0.001$; IP injection $x$ stimulation intensity: $F(1,4)=537.0, p<0.001)$ and reduced PPR (Fig 3E, saline: $1.34 \pm 0.1, n=5 ; \mathrm{KA}: 0.98$ $\pm 0.1, n=7$; saline vs KA: $p>0.05$, unpaired t-test) and CV (Fig $3 E$, saline: $0.39 \pm 0.04, n=5$; $\mathrm{KA}: 0.27 \pm 0.03, \mathrm{n}=7$; saline vs KA: $\mathrm{p}<0.05$, unpaired t-test) in mCherry+ control GCs. Of note, input/output function, PPR and CV in cKO (Cre-mCherry+) GCs and control (mCherry+) GCs after saline injection were comparable (Fig 3B-E). GC membrane properties were similar between all the different groups (Fig S1). These data suggest that while Bdnf cKO had no impact on basal MC-GC synaptic properties and GC membrane properties, it abolished seizure-induced strengthening of MC-GC synaptic transmission.

Because PKA activity is required for MC-GC LTP downstream of BDNF/TrkB signaling ${ }^{24}$ (Berthoux et al.,), we examined whether cAMP/PKA activation could still induce LTP in BDNF-deficient GCs. Bath application of forskolin $(50 \mu \mathrm{M}, 10 \mathrm{~min})$ triggered normal LTP in BDNF-deficient GCs (CremCherry+) obtained from both KA- and saline-injected mice in postsynaptic Bdnf cKOs (Fig 3F, saline: $162.9 \pm 9.4 \%$ of baseline, $n=6, p<0.01$, paired t-test; KA: $161.9 \pm 5.7 \%$ of baseline, $n$ $=5, p<0.001$, paired t-test; saline vs KA: $p>0.05$, unpaired $t$-test), supporting the idea that postsynaptic Bdnf deletion prevented KA-injection from inducing MC-GC LTP in vivo. In contrast, and consistent with our previous results (Fig 2l), forskolin failed to induce LTP in control GCs (mCherry+) obtained from KA-injected mice but not from saline-injected mice (Fig 3G, saline: $171.5 \pm 7.2 \%$ of baseline, $n=5, p<0.001$, paired t-test; KA: $115.7 \pm 8.1 \%$ of baseline, $n=7, p$ $<0.05$, paired t-test; saline vs KA: $p<0.001$, unpaired t-test). Altogether, these results reveal that initial seizures triggered MC-GC LTP in vivo via a BDNF-dependent mechanism, and that such synaptic potentiation occluded subsequent induction of LTP in vitro.

Deleting Bdnf from DG excitatory neurons, a manipulation that abolishes MC-GC LTP both in vitro (Berthoux et al) or in vivo (Fig 3), could inhibit seizure induction. To test this possibility, we stereotaxically injected AAV-CaMKII-Cre-mCherry (cKO) or AAV-CaMKII-mCherry (control) into both dorsal and ventral DG of adult Bdnfl/fil mice, bilaterally (Fig 4A). We confirmed that the virus was highly expressed in the DG (Fig 4B) and found that at least $70 \%$ of DG neurons were mCherry+. Bdnf conditional deletion reduced seizure severity and susceptibility, as indicated by significant decrease in the total cumulative seizure score (Fig 4C, two way ANOVA RM, AAV 
condition: $F(1,7)=6.0, p<0.05$, time: $F(1.26,8.87)=150.4, p<0.001 ;$ AAV condition $x$ time: $\mathrm{F}(1,7)=262.4, \mathrm{p}<0.001$ ), in sum score (Fig 4D, control: $48.9 \pm 6.0, \mathrm{n}=8$; $\mathrm{cKO}: 31.7 \pm 4.7, \mathrm{n}=$ 9; control vs cKO: $p<0.05$, unpaired t-test) and increase in latency to stage 3 (Fig 4E, control: $21.2 \pm 3.5, \mathrm{n}=8$; cKO: $51.1 \pm 13.4, \mathrm{n}=9$; control vs cKO: $\mathrm{p}<0.05$, Mann Whitney test), respectively. Remarkably, c-Fos expression was significantly reduced in Bdnf cKO mice as compared to controls (Fig S2). These results indicate that seizure-induced MC-CG LTP may have a pro-convulsant action.

\section{In vivo application of MC-GC LTP induction protocol promoted epileptic seizures}

We previously showed that optogenetic repetitive stimulation of MC axons triggers robust MC-GC LTP in vitro ${ }^{24}$. We therefore tested whether in vivo induction of LTP using optogenetic activation of MCs promotes seizure activity. To this end, we selectively expressed ChiEF in MCs by bilaterally injecting the Cre-recombinase-dependent AAV-hSyn-DIO-ChIEF-Tdtomato into the dorsal and ventral DG of Drd2-Cre mice (Fig 5A). Blue light stimulation (MC BS: 5 pulses of $5 \mathrm{~ms}$ at $30 \mathrm{~Hz}$, repeated 50 times every $0.5 \mathrm{~s}$ ) was delivered through an optic fiber placed above the dorsal DG IML in vivo and sham-light was used as a control (Fig 5A, B and F). Optic fiber location and selective viral expression in MCs were confirmed posthoc (Fig 5B). We also verified that the light stimulation protocol induced MC-GC LTP in vivo. If so, this plasticity should be occluded in hippocampal slices prepared after in vivo light stimulation. We found that repetitive light stimulation of MC axons failed to induce LTP in vitro when LTP protocol was pre-applied in vivo as compared to control slices (Fig 5C, after in vivo LTP: $96.1 \pm 7.9 \%$ of baseline, $n=6, p>0.05$, paired t-test; control: $146.7 \pm 4.9 \%$ of baseline, $n=5, p<0.001$, paired t-test; in vivo LTP vs control: $p<0.001$, unpaired t-test). In addition, both PPR (Fig 5D, control: $1.51 \pm 0.10, n=5$; in vivo LTP: $1.08 \pm 0.09, n=6$; control vs in vivo LTP: $p<0.05$, unpaired t-test) and CV (Fig 5E, control: $0.41 \pm 0.04, n=5$; in vivo LTP: $0.25 \pm 0.04, n=6$; control vs in vivo LTP: $p<0.05$, unpaired t-test) were significantly reduced after in vivo photo-stimulation as compared to control mice (sham light). These results strongly suggest that in vivo light stimulation of MC axons (MC BS: 5 pulses of $5 \mathrm{~ms}$ at $30 \mathrm{~Hz}$, repeated 50 times every $0.5 \mathrm{~s}$ ) induced presynaptic LTP at MC-GC synapses. We then tested whether in vivo induction of MC-GC LTP can promote epileptic seizures. Seizures were induced with $20 \mathrm{mg} / \mathrm{kg} \mathrm{KA} \mathrm{IP,} 50 \mathrm{~min}$ after in vivo induction of LTP (Fig $5 \mathrm{~F}$ ). Light delivery alone was not sufficient to trigger any behavioral seizure (data not shown). Remarkably, we found that in vivo application of a MC-GC LTP induction protocol significantly increased the severity and susceptibility of convulsive seizures induced by KA, as indicated, 
respectively, by significant increase in cumulative seizure score (Fig 5G, two way ANOVA RM, light: $F(1,3)=100.7, p<0.001$, time: $F(1.3,4.1)=221.5, p<0.001$; light $x$ time: $F(1,3)=109.3$, $\mathrm{p}<0.01$ ), in sum score (Fig $5 \mathrm{H}$, control: $18.0 \pm 1.8, \mathrm{n}=5$; in vivo LTP: $28.0 \pm 1.5, \mathrm{n}=4$; control vs in vivo LTP: $p<0.01$, unpaired t-test), and a significantly decrease in latency to convulsive seizures (Fig 5I, control: $104.0 \pm 10.3, \mathrm{n}=5$; in vivo LTP: $42.5 \pm 7.5, \mathrm{n}=4$; control vs in vivo LTP: $p<0.05$, Mann Whitney test). While optogenetic activation of MCs could impact their basic membrane properties or synaptic inputs, we found that the stimulation protocol that triggers LTP in vivo ( $5 \mathrm{MCs}$ action potential at $30 \mathrm{~Hz}$, repeated 50 times every $0.5 \mathrm{~s}$ ) did not significantly alter MC membrane resistance nor the main excitatory synaptic drive, i.e., GC inputs, onto MC recorded in vitro (Fig S3) (see Methods). Taken together, these findings strongly suggest that in vivo induction of MC-GC LTP can worsen epileptic seizures.

\section{DISCUSSION}

In this study, we found that early seizures potentiate crucial hippocampal excitatory synapses, thereby facilitating further epileptic activity. KA-induced acute epileptic seizures, not only increased MC and GC activity in vivo, but also triggered a BDNF-dependent strengthening of MCGC synaptic transmission that occluded subsequent induction of MC-GC LTP. In addition, blocking MC-GC LTP and silencing MC selectively, were both associated with significant decrease in seizure susceptibility and severity. Moreover, in vivo induction of MC-GC LTP was sufficient to worsen convulsive seizures subsequently triggered with KA. Overall, our findings strongly suggest that seizure-induced plasticity at MC-GC excitatory synapses may promote epilepsy and significantly contribute to the pro-convulsant role of MCs during early stages of epilepsy.

\section{Initial seizures induced BDNF-dependent strengthening of MC-GC synaptic transmission}

Using in vivo 2-photon live imaging in awake behaving mice, we found that acutely induced seizures triggered a massive increase in both MC and GC calcium signal (Fig 2), indicating a robust increase in neuronal activity. Our findings are consistent with a recent study reporting in vivo epileptiform calcium signals detected with fiber photometry in the DG following KA administration ${ }^{35}$. While these signals likely reflect the activity of a large population of neurons, including interneurons, we could assess calcium activity of individual MCs and GCs by combining selective expression of a calcium indicator in DG excitatory neurons and 2-photon live imaging. It 
has been reported that dorsal GCs are mainly activated by ventral $\mathrm{MCs}^{34}$. However, because of the limited access to the ventral hippocampus, 2-photon live imaging did not allow us to directly assess which of MCs or GCs take the lead during epileptic activity. We hypothesize that KA administration activates MCs which in turn engage GCs. Consistent with this idea, we have recently found that MCs express functional extrasynaptic kainate receptors whose activation with sub-micromolar concentrations of KA can drive MC activity in vitro; whereas GCs show comparatively much less sensitivity (at least one order of magnitude) to KA application ${ }^{38}$. Although interneurons ${ }^{39}$ and CA3 pyramidal cells also express kainate receptors ${ }^{40,41}$, it is unlikely that activation of these neurons could directly drive GCs. Furthermore, MCs show higher activity in vivo in contrast to $\mathrm{GCs}^{42-44}$, making them more likely to be engaged during epileptic activity, independently of the nature of the chemoconvulsant. This last notion is also supported by the fact that MC silencing not only reduced KA-induced seizures (Fig 1) but also prevents pilocarpineinduced epilepsy ${ }^{5}$. Although MCs also excite inhibitory interneurons ${ }^{17}$, the anti-convulsant effect of $\mathrm{MC}$ silencing (Fig 1) suggests that MC silencing during initial seizures has a stronger impact on the activity of GCs than interneurons ${ }^{5}$. Besides inducing synaptic plasticity, KA-induced increase in $\mathrm{MC}$ and $\mathrm{GC}$ intracellular calcium concentration (Fig 2) may also contribute to excitotoxicity and cell death. Altogether, our findings demonstrate that MCs and GCs are highly active during initial experimental seizures, suggesting that sustained activation of MCs contributed to GC recruitment.

We gathered multiple lines of evidence indicating that initial convulsive seizures induced presynaptically expressed MC-GC LTP in vivo. MC-GC synaptic strength was increased in KAtreated mice as compared to sham-injected animals, and this strengthening was associated with a significant reduction in both PPR and CV (Fig 2), suggesting a presynaptic mechanism. Furthermore, induction of MC-GC LTP in vitro was occluded after convulsive seizures (Fig 2), indicating a common step. The KA-induced strengthening of MC-GC synaptic transmission in vivo was likely induced by the increase in $\mathrm{MC}$ activity ${ }^{38}$, consistent with the observation that repetitive MC activity triggers robust MC-GC LTP in acute rodent hippocampal slices ${ }^{24}$. Although in vitro epileptic activity was associated with a rise in the net excitatory drive between MCs and GCs ${ }^{5}$, it is unclear whether this effect results from disinhibition or direct MC-GC synaptic strengthening. Our findings show that both in vivo optogenetic activation of MCs (Fig 5) and acute seizures (Fig 2) were sufficient to trigger presynaptic MC-GC LTP. 
Several studies indicate that seizures can increase both BDNF levels ${ }^{45-48}$ and TrkB activation in the hippocampus ${ }^{26,49}$. In addition, BDNF is necessary and sufficient for MC-GC LTP ${ }^{24}$, and it can be released from both MCs and GCs following MC repetitive activity in vitro ${ }^{25}$. It is therefore likely that by releasing BDNF, MC and GC activity induces MC-GC LTP in vivo. In support of this mechanism, we found that genetic removal of Bdnf from GCs abolished seizure-induced MC-GC LTP (Fig 3), while it did not affect basal MC-GC synaptic (Fig 3) nor GC membrane properties in sham-injected mice (Fig S1). Of note, we did not observe any failure of seizure induction when Bdnf was sparsely $\mathrm{KO}$. Altogether, our new findings indicate that BDNF mediates in vivo seizureinduced strengthening of MC-GC excitatory synapses.

\section{Seizure-induced LTP at MC-GC synapse promotes epilepsy}

Our findings strongly suggest that activity-dependent strengthening of MC-GC synapses promotes acute seizures. While MCs innervate GCs and inhibitory interneurons, MC repetitive activity that induces MC-GC LTP, at least in vitro, has no effect on feed-forward inhibition onto $\mathrm{GCs}^{24}$, and such repetitive activity does not induce plasticity at GC-MC synapses either (Fig S3). LTP-induced worsening of seizures (Fig 5 ) is supported by the extensive MC projection onto the proximal dendrites of $\mathrm{GCs}^{17}$ and the powerful MC-GC excitatory drive reported in vitro ${ }^{24}$ and in vivo $^{34}$. Given that a single MC innervates as much as $75 \%$ of the septotemporal axis of the hippocampus ${ }^{50}$, broad induction of MC-GC LTP can be detrimental, underscoring a link between uncontrolled LTP at hippocampal excitatory synapse and seizures. Conversely, blocking activitydependent strengthening of MC-GC synapses in vivo reduced seizures. Protein kinase A (PKA) and BDNF signaling pathways are both necessary and sufficient for activity-dependent LTP at MC-GC synapses ${ }^{24,25}$. MC silencing using G $_{i}$ inhibitory DREADD (Fig 1), and knocking out Bdnf from hippocampal excitatory neurons (Fig 4) both reduced acute KA-induced seizures. However, MC silencing not only prevents MC-GC LTP induction but also reduces basal MC-GC synaptic transmission (Fig 1) and MC output activity. Although Bdnf cKO had no effect on MC-GC basal transmission (Fig 3) and GC excitability (Fig S1), we cannot discard an effect on other synapses including GC-CA3 synaptic transmission and plasticity. Bdnf deletion reduced c-Fos expression in the DG of KA-injected mice (Fig S2), suggesting that BDNF/TrkB signaling contributes to seizure-induced opening of the DG gate likely by strengthening MC-GC synapses. Consistent with this scenario, optogenetic induction of MC-GC LTP in vivo was sufficient to worsen convulsive seizures. Notably, type-1 cannabinoid receptors, which are highly expressed at MC terminals ${ }^{51}$, tonically suppress MC-GC transmission and also dampen the induction of MC-GC 
LTP $^{52}$ in an activity-dependent manner. By suppressing excitatory drive, these receptors could be a potential target to prevent epilepsy ${ }^{53-55}$. In agreement with recent findings using the pilocarpinemodel $^{5}$, our results strongly support a pro-convulsant role of MCs during early epilepsy. In contrast, in a chronic mouse model of TLE induced by KA intrahippocampal administration, MCs are reportedly anti-epileptic ${ }^{4}$, suggesting that the role of MCs may differ significantly with the disease stage.

Compelling evidence indicates that BDNF and its high-affinity receptor TrkB promote worsening of TLE ${ }^{26,27,36,37,56-60}$, but the precise mechanisms and specific contribution of different cell types are not entirely clear. Here we identified two reciprocally connected excitatory neurons in the dentate gyrus, MCs and GCs, that can mediate the BDNF pro-convulsant effects via BDNFdependent MC-GC LTP. Interfering with BDNF/TrkB signaling in different ways reduced epilepsy --i.e. heterozygous deletion of $B d n f^{36}$, neuronal deletion of $B d n f$ or $T r k B^{37}$, chemogenetic blockade of TrkB kinase activity in $\operatorname{TrkB}^{\mathrm{F} 616 \mathrm{~A}}$ mutant mice ${ }^{59}$ and a mutant mouse that uncouples TrkB from its downstream phospholipase Cy1 signaling ${ }^{57}$. Importantly, based on our previous ${ }^{24}$ and present findings (Fig 3,5), these manipulations could also prevent seizure-induced MC-GC LTP and the associated facilitation of epileptic activity. Conversely, overexpression of BDNF in the brain ${ }^{60}$ and local infusion of BDNF into the hippocampus ${ }^{56}$ worsen epileptic seizures. Because BDNF is sufficient to induce MC-GC LTP in vitro ${ }^{24}$, it is likely that in vivo infusion of BDNF promotes seizures by inducing MC-GC LTP broadly. Of note, exogenous BDNF delivery into the hippocampus of chronically epileptic rats can have anti-epileptic and neuroprotective effects ${ }^{61}$, suggesting that BDNF action might differ with epilepsy stages.

Altogether our findings uncover a potential mechanism implicated in the early stages of epilepsy, before the brain damages become irreversible. We highlighted how initial seizures can shape an important but overlooked hippocampal excitatory synapses in a BDNF-dependent manner, and how broad, uncontrolled induction of LTP can be detrimental and promote subsequent induction of seizures. Manipulations that suppress LTP induction and BDNF signaling at MC-GC synapses may be a new strategy for the treatment of epilepsy.

\section{METHODS}

\section{Experimental Model and Subject Details}

C57BL/6, Bdnf floxed (Bdnff/flf) or Drd2-cre (B6.FVB(Cg)-Tg(Drd2-cre)ER44Gsat/Mmucd, MMRRC 032108-UCD) mice (2-3.5-month-old, both males and females) were used in this study. 
All animals were group housed in a standard $12 \mathrm{hr}$ light/12 hr dark cycle and had free access to food and water. Animal handling, breeding and use followed a protocol approved by the Animal Care and Use Committee of Albert Einstein College of Medicine, in accordance with the National Institutes of Health guidelines. Bdnfl/fl mice, generated by Dr. Jaenisch, were kindly donated by Dr. Lisa Monteggia (University of Texas, Southwestern Medical Center).

\section{Acute hippocampal slice preparation}

Acute transverse hippocampal slices (300 $\mu \mathrm{m}$ thick) were prepared from C57BL/6, Bdnf fl/fl and Drd2-cre mice. Animals were anesthetized with isoflurane and euthanized in accordance with institutional regulations. The hippocampi were then removed and cut using a VT1200s Microslicer (Leica Microsystems Co.) in a solution containing (in mM): $93 \mathrm{~N}$-methyl-D-glucamine (NMDG), $2.5 \mathrm{KCl}, 1.25 \mathrm{NaH}_{2} \mathrm{PO}_{4}, 30 \mathrm{NaHCO}_{3}, 20$ HEPES, 25 D-glucose, 2 Thiourea, 5 Na-Ascorbate, 3 Na-Pyruvate, $0.5 \mathrm{CaCl}_{2}, 10 \mathrm{MgCl}_{2}, 93 \mathrm{HCl}$. These slices were then transferred to $32^{\circ} \mathrm{C}$ extracellular artificial cerebrospinal fluid (ACSF) solution, containing (in $\mathrm{mM}$ ): $124 \mathrm{NaCl}, 2.5 \mathrm{KCl}$, $26 \mathrm{NaHCO}_{3}, 1 \mathrm{NaH}_{2} \mathrm{PO}_{4}, 2.5 \mathrm{CaCl}_{2}, 1.3 \mathrm{MgSO}_{4}$ and $10 \mathrm{D}$-glucose, for 30 min and then kept at room temperature for at least 40 min before recording. All solutions were equilibrated with $95 \%$ $\mathrm{O}_{2}$ and $5 \% \mathrm{CO}_{2}(\mathrm{pH} 7.4)$.

\section{Electrophysiology}

All recordings were performed at $28 \pm 1^{\circ} \mathrm{C}$ in a submersion-type recording chamber perfused at $2 \mathrm{ml} / \mathrm{min}$ with $\mathrm{ACSF}$. GABA $\mathrm{GA}_{\mathrm{A}}$ and $\mathrm{GABA}_{B}$ receptor antagonists, picrotoxin $(100 \mu \mathrm{M})$ and CGP55845 hydrochloride ( $3 \mu \mathrm{M})$, were included in the extracellular solution (ACSF) except in the experiments shown in Fig S3. Whole-cell patch-clamp recordings using a Multiclamp 700A amplifier (Molecular Devices) were made from GCs (in the upper blade of the DG) and MCs (Fig S3) voltage clamped at $-60 \mathrm{mV}\left(\mathrm{V}_{\mathrm{h}}=-60 \mathrm{mV}\right)$ using patch-type pipette electrodes $(\sim 3-4 \mathrm{M} \Omega)$ containing (in mM): $135 \mathrm{KMeSO}_{4}, 5 \mathrm{KCl}, 1 \mathrm{CaCl}_{2}, 5 \mathrm{NaOH}, 10 \mathrm{HEPES}, 5 \mathrm{MgATP}, 0.4 \mathrm{Na}_{3} \mathrm{GTP}, 5$ EGTA and 10 D-glucose, pH 7.2 (288-291 mOsm). Series resistance ( $\sim 6-25 \mathrm{M} \Omega$ for GCs and $\sim 17-$ $21 \mathrm{M} \Omega$ for MCs) was monitored throughout all experiments with a $-5 \mathrm{mV}, 80 \mathrm{~ms}$ voltage step, and cells that exhibited a significant change in series resistance ( $>20 \%)$ were excluded from analysis. Mature GCs were identified by characteristic hyperpolarized membrane potential (checked immediately after membrane break in, -72 to $-83 \mathrm{mV}$ ). Cells with high membrane resistance (> $800 \mathrm{M} \Omega$ ) were considered as putative adult born $\mathrm{GCs}^{62}$ and were excluded from the analysis. To activate MC axons, a broken tip ( 10-20 $\mu \mathrm{m})$ stimulating patch-type micropipette filled with ACSF was placed in the IML ( $<50 \mu \mathrm{m}$ from the border of the GC body layer) and paired, monopolar square-wave voltage or current pulses (100 $\mu$ s pulse width, 4-30 V) were delivered through a stimulus isolator (Digitimer DS2A-MKII). Typically, stimulation intensity was adjusted to obtain comparable magnitude of synaptic responses across experiments, e.g., 30-80 pA EPSCs $\left(V_{h}=\right.$ $-60 \mathrm{mV}$ ), except for input/output experiments shown in Fig 2F, 3B and 3D. MC-GC LTP was typically induced by 5 stimuli at $100 \mathrm{~Hz}$ repeated 50 times, every $0.5 \mathrm{~s}$, except in Fig 5 (see below). Light evoked EPSCs (o-EPSCs), shown in Fig $5 \mathrm{C}-\mathrm{E}$, were triggered using $1 \mathrm{~ms}$ pulses of blue light, provided by a collimated LED (Thorlabs, M470L3-C5, $470 \mathrm{~nm}, 300 \mathrm{~mW}$ ) and delivered through the microscope objective (40X, $0.8 \mathrm{NA})$. Recordings were performed in acute hippocampal slices $<700 \mu \mathrm{m}$ from the optic fiber implant. The light was centered in the IML, and the light intensity adjusted to obtain $\sim 60-120 \mathrm{pA}$ responses $\left(\mathrm{V}_{\mathrm{h}}=-60 \mathrm{mV}\right)$. In the experiments shown in Fig 5C, LTP was induced using light stimulation of $\mathrm{MC}$ axons with the following protocol: 5 pulses of $1 \mathrm{~ms}$, at $30 \mathrm{~Hz}$ repeated 50 times, every $0.5 \mathrm{~s}$. In Fig S3, GC-MC synaptic strength was monitored. MCs identity was confirmed by the presence of a characteristic high frequency of spontaneous EPSCs, and by checking the firing pattern in response to depolarizing step of currents (non-burst firing and action potentials with almost no afterhyperpolarization) ${ }^{63}$. GC axons were electrically stimulated in the DG subgranular zone using a bipolar theta glass ACSF-filled 
pipette. The mGluR2/3 agonist DCG-IV $(1 \mu \mathrm{M})$, which selectively reduces GC-MC synaptic transmission ${ }^{64,65}$ was applied at the end of each experiment to confirm the nature of the stimulated input. To mimic physiological conditions, EPSCs were recorded in absence of GABA receptor blockers. Reagents were bath applied following dilution into ACSF from stock solutions stored at $-20^{\circ} \mathrm{C}$ prepared in water or DMSO, depending on the manufacturer's recommendation.

\section{BDNF conditional KO}

$B d n f^{f / f l}$ mice were injected with Cre expressing (AAV5-CamKII-Cre-mCherry, $5.8 \times 10^{12}$ Virus Molecules $/ \mathrm{mL}$, UNC Vector) or control (AAV5-CamKII-mCherry, $4.9 \times 10^{12}$ Virus Molecules $/ \mathrm{mL}$, UNC Vector) adeno-associated viruses. For electrophysiological experiments, $0.5 \mu$ l of virus was injected (flow rate of $0.1 \mu \mathrm{l} / \mathrm{min}$ ) unilaterally into the dorsal blade of the DG (relative to bregma: $2.06 \mathrm{~mm}$ posterior, $1.5 \mathrm{~mm}$ lateral, $1.8 \mathrm{~mm}$ ventral) of 5-8-week-old (w.o.) Bdnfl/fl mice. Slices for electrophysiology were prepared from injected animals, 2 to 4 weeks after injection. In postsynaptic cKO animals, we verified the absence of mCherry in the hilus of the whole ipsilateral hippocampus, as previously described ${ }^{24}$. For seizure monitoring and c-Fos labeling experiments shown in Fig 4 and Fig S2, $0.5 \mu \mathrm{l}$ of virus was injected (flow rate of $0.1 \mu \mathrm{l} / \mathrm{min}$ ) bilaterally into both the dorsal (relative to bregma: $1.9 \mathrm{~mm}$ posterior, $1.25 \mathrm{~mm}$ lateral, $2.1 \mathrm{~mm}$ ventral) and ventral (relative to bregma: $3.2 \mathrm{~mm}$ posterior, $2.2 \mathrm{~mm}$ lateral, $2.8 \mathrm{~mm}$ ventral) DG of 5-8-w.o. Bdnf $f^{l / f l}$ mice and seizure was induced and monitored 2-3 weeks post-injection. Animals were placed in a stereotaxic frame and anesthetized with isoflurane (up to $5 \%$ for induction and $1 \%-3 \%$ for maintenance). In all experiments, both male and female mice were used with a similar ratio for the two types of viruses.

\section{MC silencing}

To silence MCs, the Gi inhibitory DREADD (iDREADD) was selectively expressed in MCs. The Cre-dependent AAV-CamKII-DIO-hM4D $\left(\mathrm{G}_{\mathrm{i}}\right)$-mCherry $\left(4.16 \times 10^{13} \mathrm{vg} / \mathrm{mL}\right.$, prepared at Janelia Research Campus) or AAV-CamKII-DIO-mCherry (control, $5.95 \times 10^{13} \mathrm{vg} / \mathrm{mL}$, prepared at Janelia Research Campus) was injected $(0.5 \mu \mathrm{l} / \mathrm{site}$, at $0.1 \mu \mathrm{l} / \mathrm{min}$ ) bilaterally into both the dorsal (relative to bregma: $1.9 \mathrm{~mm}$ posterior, $1.25 \mathrm{~mm}$ lateral, $2.1 \mathrm{~mm}$ ventral) and ventral (relative to bregma: $3.2 \mathrm{~mm}$ posterior, $2.2 \mathrm{~mm}$ lateral, $2.8 \mathrm{~mm}$ ventral) DG of 5-8-w.o. Drd2-Cre mice. Seizures were induced and monitored 2-3 weeks post-injection. The DREADD selective agonist clozapine-Noxide (CNO, TOCRIS) was bath applied in acute slices in experiments shown in Fig 1D. CNO was administered intraperitoneally in Fig $1 \mathrm{E}-\mathrm{H}(2 \mathrm{mg} / \mathrm{kg}$ diluted in saline solution containing $2 \%$ DMSO) 30 min before in vivo seizure induction (KA IP), based on kinetics of CNO plasma levels and in vivo effects ${ }^{66,67}$. Viral expression was verified posthoc.

\section{In vivo induction of MC-GC LTP with optogenetics}

To induce MC-GC LTP in vivo by light-activating MCs, ChiEF was selectively expressed in MCs, and an optic fiber (200 $\mu \mathrm{m}$ diameter) was implanted unilaterally (relative to bregma: $1.9 \mathrm{~mm}$ posterior, $1.25 \mathrm{~mm}$ lateral, $1.8 \mathrm{~mm}$ ventral) to deliver blue light above the IML of the DG. The Credependent AAV-hSyn-Flex-ChIEF-Tdtomato $\left(2.4 \times 10^{12}\right.$ Virus Molecules $\left./ \mathrm{mL}\right)$ was injected bilaterally $(0.5 \mu \mathrm{l} / \mathrm{site}$, at $0.1 \mu \mathrm{l} / \mathrm{min})$ into both the dorsal (relative to bregma: $1.9 \mathrm{~mm}$ posterior, $1.25 \mathrm{~mm}$ lateral, $2.1 \mathrm{~mm}$ ventral) and ventral (relative to bregma: $3.2 \mathrm{~mm}$ posterior, $2.2 \mathrm{~mm}$ lateral, $2.8 \mathrm{~mm}$ ventral) DG of 5-8-w.o. Drd2-Cre mice. 5-7 weeks after surgery, MC-GC LTP induction protocol $^{24}$ was applied in vivo, by delivering a brief burst of blue light ( 5 pulses of $5 \mathrm{~ms}$ at $30 \mathrm{~Hz}$, repeated 50 times every $0.5 \mathrm{~s}$ ), using a fiber-coupled $470 \mathrm{~nm}$ LED light source (Thorlabs, MF470F3, $7 \mathrm{~mW}$ output from the $200 \mu \mathrm{m}$ optic fiber). Viral expression and optic fiber implant location were confirmed posthoc.

\section{Seizure induction and monitoring}


Seizures were induced acutely using intraperitoneal (IP) injection of $20-30 \mathrm{mg} / \mathrm{kg}$ of kainic acid (KA, HelloBio HB0355), prepared in saline solution the same day, in 2-3-month-old mice. To be able to see potential decrease and increase in seizure severity/susceptibility, $30 \mathrm{mg} / \mathrm{kg}$ (Fig 1 and 4) and $20 \mathrm{mg} / \mathrm{kg}$ (Fig 5) of KA were used, respectively. Of note, seizures were less severe in Drd2-Cre mice as compared to C57BL/6 (wild type) and Bdnfl/fl animals, likely due to strain differences $^{33}$. For MC-GC basal transmission and synaptic plasticity analysis, acute hippocampal slices were prepared when the animal reached stage 3 of convulsive seizures, i.e., forelimb clonus and rearing (Fig 2E-I) or 25 min post KA IP injections (Fig 3). Saline-injected mice were used as a control. For behavioral seizure scoring, mice were monitored during 120 min post-injection and behavioral seizures were scored, by an experimenter blind to condition (control vs Bdnf cKO or control vs MC silencing), using a modified Racine scale ${ }^{68}$ as follows: stage 0: normal behavior, stage 1: immobility and rigidity, stage 2 : head bobbing, stage 3 : forelimb clonus and rearing, stage 4: continuous rearing and falling, stage 5: clonic-tonic seizure, stage 6: death. The maximum Racine score was recorded every 10 minutes and the cumulative seizure score was obtained by summing these scores across all 12 bins of the 120 min experiment. Mouse movements were visualized with an infrared camera for $2 \mathrm{P}$ imaging experiments (see below). For $\mathrm{MC}$ silencing experiments, IP injection of CNO (2 mg/kg, I.P) was delivered $30 \mathrm{~min}$ before inducing seizures with $30 \mathrm{mg} / \mathrm{kg} \mathrm{KA} \mathrm{IP} \mathrm{injection} \mathrm{to} \mathrm{both} \mathrm{groups,} \mathrm{control} \mathrm{and} \mathrm{inhibitory} \mathrm{DREADD.} \mathrm{For} \mathrm{in} \mathrm{vivo}$ optogenetic experiments, LTP was induced 50 min before KA IP injection, and animals receiving sham-light stimulation were used as control.

\section{c-Fos immunolabeling and posthoc confirmation of AAV expression}

Two hours after KA (20-30 mg/kg in saline) intraperitoneal injection, mice were deeply anesthetized using isoflurane (3-5\%) and transcardially perfused with $4 \%$ paraformaldehyde (PFA) in $0.1 \mathrm{M}$ sodium phosphate buffer (PBS). After 24-48h fixation in 4\% PFA, $50 \mu \mathrm{m}$-thick brain coronal sections were prepared using a DSK Microslicer (DTK-1000). Brain slices were washed in PBS ( $3 \times 10 \mathrm{~min})$ and then incubated for $2 \mathrm{~h}$ at room temperature in a blocking solution containing $10 \%$ goat serum, $1 \%$ Triton X-100 in PBS. Primary antibody was applied for $24 \mathrm{~h}$, at $4^{\circ} \mathrm{C}$ (rabbit anti-c-Fos, 1:1000; Cell Signaling, cat \# 2250S, 0.1\% Triton X-100, 5\% goat in PBS). After 4 washes of 10 min in PBS, the sections were incubated with AlexaFluor 488-conjugated goat anti-rabbit (1:500, Invitrogen, cat \# A11008) for $2 \mathrm{~h}$ at room temperature. Slices were washed twice in PBS for 10 min each, stained with 40,6-diamidino-2-phenylindole (DAPI, 1:1000 in PBS, $20 \mathrm{~min}$, ThermoFisher) to label cell nuclei and mounted with Prolong diamond antifade reagent mountant (ThermoFisher) onto microscope slides.

\section{Image Acquisition and cell quantification}

Images were acquired using a Zeiss LSM 880 Airyscan Confocal microscope with SuperResolution and ZEN (black edition) software and a 25X oil-immersion objective. All images were analyzed using Fiji and cells were counted using Cell Counter plug-in in Fiji, in the upper and lower blade of the DG in areas of maximum viral expression (> $70 \%$ of mCherry+ neurons), by an experimenter blind to the conditions. All infected cells were confirmed by using the DAPI channel, and individual counts were taken for c-Fos-expressing neuron to provide a percentage of c-Fos+ cells among the total number of neurons. For each animal, we used 1 dorsal and 1 ventral section. Right and left hippocampus were randomly used.

\section{Adeno-Associated Virus Vector Construction}

For the construction of AAV plasmids, the human Synapsin (hSyn) promoter in pAAV-hSyn-DIOhM4D(Gi)-mCherry (Addgene, cat \#: 50459) was replaced with mouse CaMKIla promoter using Mlul and Sall restriction sites to produce pAAV-CaMKII-DIO-hM4D(Gi)-mCherry and pAAVCaMKII-DIO-mCherry. The AAVs were prepared at Janelia Research Campus. 


\section{Data analysis}

Electrophysiological data were acquired at $5 \mathrm{kHz}$, filtered at $2.4 \mathrm{kHz}$, and analyzed using a Multiclamp 700A amplifier (Molecular Devices) and custom-made software for IgorPro 7.01 (Wavemetrics Inc.). PPR was defined as the ratio of the amplitude of the second EPSC (baseline taken 1-2 ms before the stimulus artifact) to the amplitude of the first EPSC. The interval between first and second EPSC was $100 \mathrm{~ms}$. CV was calculated as the standard deviation of EPSC amplitude divided by mean EPSC amplitude. For each experiment, an average of 30 consecutive PPRs and CVs were used. The magnitude of LTP was determined by comparing 10 min baseline responses with responses 20-30 min after induction protocol. Averaged traces include 20 consecutive individual responses (or 5 consecutive responses for input/output experiments in Figures 2F, 3B and 3D).

\section{In vivo 2-photon imaging}

WT mice (6-8-w.o.) were injected in the right dorsal hippocampus (relative to bregma: $1.2 \mathrm{~mm}$ posterior, $1.6 \mathrm{~mm}$ lateral, $1.9 \mathrm{~mm}$ ventral) with a viral vector encoding for the red-shifted $\mathrm{Ca}^{2+}$ sensor jRGECO1a [AAVDJ-CaMKIla::NES-jRGECO1a-WPRE-SV40 (AAV-CaMKII-jRGECO1a), $950 \mu \mathrm{L}$ at $5.4 \times 10^{12}$ viral particles/mL, University of North Carolina Vector Core, plasmid kindly donated by Dr. Fred Gage]. A 3-mm diameter craniotomy was drilled around the injection site 24 hrs after viral injection ${ }^{69}$, the meninges, cortex, and corpus callosum were removed by aspiration and the hippocampus and alveus fibers were left intact. The lesion was irrigated with sterile saline throughout the procedure. A titanium window (3-mm diameter, 1.3-mm deep) with a glass bottom was implanted above the hippocampus. The implant was secured using dental cement and a titanium bar $(34 \times 4 \times 1.3 \mathrm{~mm})$ was attached to head-fix the mice to the microscope set-up. In vivo calcium imaging was performed 3-4 weeks after surgery, using a two-photon microscope (Thorlabs Bergamo) equipped with a 16x 0.8NA objective (Nikon) and a Fidelity-2 $1070 \mathrm{~nm}$ laser (Coherent). The mice were head-fixed and placed on a $180 \mathrm{~cm}$-long treadmill belt. For optimum light transmission, the angle of the mouse's head was adjusted to ensure that the imaging window was parallel to the objective. Movies of $\mathrm{Ca}^{2+}$ activity were acquired at $15 \mathrm{frames} / \mathrm{s}$ using an average laser power of $\sim 180 \mathrm{~mW}$, as measured in front of the objective.

Stage 3 convulsive seizures (forelimb clonus) were identified using an infra-red camera. Raw calcium movie data was preprocessed using Suite $2 \mathrm{p}^{70}$ applying default settings for alignment, registration, and extraction of regions of interest (ROIs). Manual curation was then performed to select significant ROIs according to morphology and activity data. Raw calcium traces and neuropil were extracted for further analysis with a custom written algorithm (Python). The neuropil was subtracted from each detected cell to remove contamination. For KA-injected mice, the mean fluorescence was determined for 500 frames selected during the onset of behavioral seizures (as determined by the observation of forelimb clonus), for each cell. For baseline activity, 500 frames were selected before IP injection. For control groups, frame selection was performed similarly before (baseline) and after saline injection, matching the time of KA injected mice. $\Delta \mathrm{F} / \mathrm{F}$ was calculated as follows for both groups, for each cell:

$$
\begin{gathered}
\Delta \mathrm{F} / \mathrm{F}_{\text {seizure }}=\frac{\text { Fseizure }- \text { Fbaseline }}{\text { Fbaseline }} \\
\Delta \mathrm{F} / \mathrm{F}_{\text {saline }}=\frac{\text { Fsaline }- \text { Fbaseline }}{\text { Fbaseline }}
\end{gathered}
$$

\section{Quantification and Statistical Analysis}

The normality of all distributions was assessed using the Shapiro-Wilk test. In normal distributions, Student's unpaired and paired two-tailed t tests were used to assess between-group and within- 
group differences, respectively. The non-parametric paired sample Wilcoxon signed rank test and Mann-Whitney's $U$ test were used in non-normal distributions. Statistical comparison in Fig 1F, $2 \mathrm{~F}, 3 \mathrm{~B}, 3 \mathrm{D}, 4 \mathrm{C}, 5 \mathrm{G}$ was performed using two-way ANOVA with repeated measure (RM) and Greenhouse-Geiser was used for correction of degrees of freedom when sphericity was not assumed. Statistical significance was set to $p<0.05$ (***indicates $p<0.001$, ** indicates $p<$ 0.01 , and $*$ indicates $p<0.05$ ). All values are reported as the mean \pm SEM. Experiments shown in Fig 1E-H, Fig 3, Fig S1-2 and Fig 4 were performed in blind manner during data acquisition and analysis. Statistical analysis was performed using OriginPro (version b9.2.272) software (OriginLab).

ACKNOWLEDGMENTS: We thank the members of the Castillo lab for constructive feedback. We also thank Pascal Kaeser (Harvard University) for sharing an AAV-hSyn-Flex-ChIEFTdTomato plasmid and Lisa Monteggia (Vanderbilt University) for sharing Bdnfl/fil mice. This research was supported by the National Institutes of Health (NIH), R01-NS113600, R01MH125772; R01 and MH125772 to P.E.C.; Fondation pour la Recherche Médicale (Postdoctoral Fellowship for a research abroad), the Fondation Bettencourt Schueller (Prix pour les Jeunes Chercheurs 2016) and the American Epilepsy Society Postdoctoral Research Fellowship (2020) to K.N; The Einstein Training Program in Stem Cell Research from the Empire State Stem Cell Fund through New York State Department of Health Contract C34874GG to M.A.F.; and a Whitehall Foundation Research Grant (2019-05-71) to J.T.G.

Author contributions: K.N. and P.E.C. designed research and wrote the manuscript; K.N. performed and analyzed all experiments except the 2P imaging experiments that were performed and analyzed by M.A.F. and immunostainings that were performed and quantified by S.P. All authors edited the manuscript.

\section{The authors declare no competing interests}




\section{FIGURE LEGENDS}

Figure 1: Chemogenetic silencing of MCs reduced acute kain acid-induced seizures (A, B) AAV expressing mCherry (control, AAV-CaMKII-DIO-mCherry) or the inhibitory DREADD hMD4;-mCherry (iDREADD, AAV-CaMKII-DIO-hMD4;-mCherry) were injected bilaterally into the ventral and dorsal DG of Drd2-cre mice. Confocal images of the DG showing how the viral expression is selective for hilar MCs. Note the dense labeling of MC axons in the inner molecular layer (IML).

(C, D) Schematic diagram illustrating the recording configuration (C). MC EPSCs were recorded from GC in response to MC axon stimulation in the IML. (D) Representative traces (left) and time course plot (right) showing that CNO significantly reduced EPSC amplitude in slices expressing the inhibitory DREADD in MCs but not in controls. Numbers in parentheses indicate the number of cells $(n)$ and the number of animals $(N)$.

(E) Representation of the experimental timeline. Viral stereotaxic injections were performed in Drd2-cre mice to express control (AAV-CaMKII-DIO-mCherry) or iDREADD in MCs (AAV-CaMKIIDIO-hMD4i-mCherry) 2-3 weeks before assessing behavioral seizures (for $120 \mathrm{~min}$ ). All animals were treated with $\mathrm{CNO}$ in vivo (2 $\mathrm{mg} / \mathrm{kg}$, IP) $30 \mathrm{~min}$ before seizures were acutely induced with single KA IP injection (30 mg/kg).

(F-H) Chemogenetic silencing of MCs reduced seizure severity and susceptibility. Scoring of seizures using a modified Racine scale for 120 min revealed a significant decrease in cumulative seizure score $(F)$, in sum score $(G)$ and a significantly increase in latency to convulsive seizures $(\mathrm{H})$ when MCs were silenced as compared with control animals. Numbers in parentheses indicate the number of animals.

${ }^{*} p<0.5$. Data are presented as mean \pm SEM.

Figure 2: Initial convulsive seizures increased DG excitatory neuron activity and MC-GC synaptic strength

(A, B) Schematic diagram showing the experimental apparatus (A). jRGECO1a-expressing MCs and GCs were imaged before and during KA (30 mg/kg)-induced convulsive seizures, in headfixed mice monitored with IR camera. Saline injections were used as control.

(C, D) Mean Z-projection image, acquired in vivo, of jRGECO1a-expressing MCs (hilus) and GCs (granule cell layer, GCL), before and after $\mathrm{KA}$ injection (C). Average $\triangle \mathrm{F} / \mathrm{F}$ of recorded $\mathrm{MCs}$ and GCs after saline or KA injection (D). Each data point corresponds to the average value per animal. 
(E) Seizures were acutely induced using KA IP (20 mg/kg). The mice were sacrificed once stage 3 of convulsive seizures was reached and MC-GC synaptic function was accessed in acute hippocampal slices. Saline-injected mice (IP) were used as control.

(F) Representative traces and summary plot showing how input/output function was increased in KA (20 mg/kg)-injected mice. Numbers in parentheses represent number of cells $(\mathrm{n})$ and the number of mice $(\mathrm{N})$.

(G) PPR and CV were both significantly decreased in KA-treated mice as compared to salineinjected mice. Numbers in parentheses represent number of cells.

$(\mathbf{H}, \mathbf{I})$ Representative traces (left) and time-course summary plots (right) showing that LTP at MCGC synapse induced by either MC BS ( 5 pulses at $100 \mathrm{~Hz}$, repeated 50 times every $0.5 \mathrm{~s}, \mathrm{H}$ ) or $50 \mu \mathrm{M}$ forskolin (FSK, I) application was impaired in KA-injected mice (20 mg/kg KA, stage 3). Numbers in parentheses indicate the number of cells $(n)$ and the number of mice $(N)$.

${ }^{*} p<0.5,{ }^{* *} p<0.01$. Data are presented as mean \pm SEM.

\section{Figure 3: Seizure-induced MC-GC synaptic strengthening required postsynaptic BDNF}

(A) Representation of the experimental timeline. Control (AAV-CaMKII-mCherry) or Creexpressing AAV (AAV-CaMKII-Cre-mCherry) were injected unilaterally into the dorsal blade of the DG of $B d n f^{f / f l}$ mice. Seizures were induced 2-3 weeks later using KA IP (30 mg/kg) and acute hippocampal slices were prepared 25 min post-injection. We then performed whole cell recordings in mCherry+ GC, in response to MC axon stimulation.

$(B, C) K A$-induced seizure failed to increase MC-GC synaptic strength in GC lacking BDNF. EPSC Amplitude (B), PPR (C) and CV (C) were similar in Cre-mCherry+ GC obtained from KA vs salineinjected mice.

(D, E) Seizure induction triggered a normal increase in MC-GC EPSC amplitude (E) and decrease in PPR (E) and CV (E) in Bdnfl/fl mice injected with a control virus (AAV-CaMKII-mCherry).

(F) Representative traces and time-course summary plots showing that bath application of forskolin (FSK, $50 \mu \mathrm{M}, 10 \mathrm{~min}$ ) induced similar LTP in KA- and saline injected mice when BDNF was KO from GC (Bdnfl/fil mice injected with AAV-CaMKII-Cre-mCherry).

(G) LTP at MC-GC synapse induced by forskolin (FSK) was impaired in KA-injected mice (20 $\mathrm{mg} / \mathrm{kg} \mathrm{KA}$ ) as compared to saline-treated mice, in Bdnfl/fl mice injected with a control virus (AAVCaMKII-mCherry).

In panels $C$ and $E$, numbers in parentheses represent number of cells. In panels $B, D, F$ and $G$, numbers in parentheses indicate the number of cells $(n)$ and mice $(N)$.

${ }^{*} p<0.05$, n.s. $p>0.05$. Data are presented as mean \pm SEM. 
Figure 4: Knocking out BDNF from hippocampal excitatory neurons reduced KA-induced seizures.

(A) AAV-CaMKII-mCherry (control) or AAV-CaMKII-Cre-mCherry (KO) was injected bilaterally into ventral and dorsal DG of $B d n f^{f / f l}$ mice.

(B) Confocal images (right) showing the viral expression in the DG.

(C-E) Deletion of BDNF from hippocampal excitatory neurons (Bdnfl/fl mice injected with AAVCaMKII-Cre-mCherry) induced a significant decrease in cumulative seizure score (C), in sum score (D) and a significantly increase in latency to convulsive seizures (E) as compared with controls (Bdnf $f^{f / f l}$ mice injected with AAV-CaMKII-mCherry).

${ }^{*} p<0.05$. Numbers in parentheses represent number of mice. Data are presented as mean \pm SEM.

\section{Figure 5: In vivo induction of MC-GC LTP promoted seizures}

(A) Diagram of the experimental timeline. To selectively photo-stimulate MC axons in vivo, AAVhSyn-ChiEF-Tdtomato was injected bilaterally into ventral and dorsal DG of Drd2-cre mice and an optical fiber was implanted above the IML of the DG. LTP induction protocol (MC BS: 5 pulses at $30 \mathrm{~Hz}$, repeated 50 times, every $0.5 \mathrm{~s}$ ) was applied in vivo by delivering blue light through a patch cord cable connected to a fiber-coupled 470 nm LED light source, 5- 7 weeks after surgery. Shamlight was used as a control. Acute hippocampal slices were prepared 20 min later and MC-GC synaptic properties were analyzed using whole cell recordings of GCs and light stimulation of MCs axons.

(B) Confocal images showing the viral expression and the fiber tract location.

(C) O-EPSCs were recorded in GC in response to MC axon photostimulation. Representative traces (light) and time-course summary plot (light) showing that in vivo application of MC BS prevented subsequent induction of LTP in vitro. Numbers in parentheses, indicate the number of cells $(n)$ and the number of mice $(N)$.

(D, E) PPR (D) and CV (E) were both significantly decreased after in vivo application of MC-GC LTP induction protocol, as compared to sham light (control) condition. Numbers in parentheses, indicate the number of cells.

(F) Diagram of the experimental timeline. LTP induction protocol (MC BS: 5 pulses at $30 \mathrm{~Hz}$, repeated 50 times, every $0.5 \mathrm{~s}$ ) was delivered in vivo while sham-light was used as a control. KA $(20 \mathrm{mg} / \mathrm{kg} \mathrm{IP}$ ) was then administered $50 \mathrm{~min}$ after the optogenetic stimulation and seizures were scored for $120 \mathrm{~min}$. 
bioRxiv preprint doi: https://doi.org/10.1101/2021.06.30.450628; this version posted June 30, 2021. The copyright holder for this preprint (which was not certified by peer review) is the author/funder. All rights reserved. No reuse allowed without permission.

(G-I) Application of MC-GC LTP induction protocol increased seizure severity and susceptibility as indicated, respectively, by significant increase in cumulative seizure score (G), in sum score $(\mathrm{H})$, and a significantly decrease in latency to convulsive seizures $(\mathrm{I})$.

${ }^{*} p<0.05,{ }^{* *} p<0.01$. Data are presented as mean \pm SEM. 


\section{REFERENCES}

1 Fisher, R. S. et al. Epileptic seizures and epilepsy: definitions proposed by the International League Against Epilepsy (ILAE) and the International Bureau for Epilepsy (IBE). Epilepsia 46, 470-472, doi:10.1111/j.0013-9580.2005.66104.x (2005).

2 Bialer, M. et al. Progress report on new antiepileptic drugs: A summary of the Thirteenth Eilat Conference on New Antiepileptic Drugs and Devices (EILAT XIII). Epilepsia 58, 181221, doi:10.1111/epi.13634 (2017).

3 Scharfman, H. E. The enigmatic mossy cell of the dentate gyrus. Nat Rev Neurosci 17, 562-575, doi:10.1038/nrn.2016.87 (2016).

$4 \quad$ Bui, A. D. et al. Dentate gyrus mossy cells control spontaneous convulsive seizures and spatial memory. Science 359, 787-790, doi:10.1126/science.aan4074 (2018).

5 Botterill, J. J. et al. An Excitatory and Epileptogenic Effect of Dentate Gyrus Mossy Cells in a Mouse Model of Epilepsy. Cell Rep 29, 2875-2889 e2876, doi:10.1016/j.celrep.2019.10.100 (2019).

6 Chang, B. S. \& Lowenstein, D. H. Epilepsy. N Engl J Med 349, 1257-1266, doi:10.1056/NEJMra022308 (2003).

7 Jinde, S. et al. Hilar mossy cell degeneration causes transient dentate granule cell hyperexcitability and impaired pattern separation. Neuron 76, 1189-1200, doi:10.1016/j.neuron.2012.10.036 (2012).

8 Scharfman, H. E. \& Myers, C. E. Hilar mossy cells of the dentate gyrus: a historical perspective. Front Neural Circuits 6, 106, doi:10.3389/fncir.2012.00106 (2012).

9 Ratzliff, A., Santhakumar, V., Howard, A. \& Soltesz, I. Mossy cells in epilepsy: rigor mortis or vigor mortis? Trends Neurosci 25, 140-144, doi:10.1016/s0166-2236(00)02122-6 (2002).

10 Ratzliff, A., Howard, A. L., Santhakumar, V., Osapay, I. \& Soltesz, I. Rapid deletion of mossy cells does not result in a hyperexcitable dentate gyrus: implications for epileptogenesis. J Neurosci 24, 2259-2269, doi:10.1523/JNEUROSCI.5191-03.2004 (2004).

11 Blumcke, I. et al. Loss of hilar mossy cells in Ammon's horn sclerosis. Epilepsia 41 Suppl 6, S174-180, doi:10.1111/j.1528-1157.2000.tb01577.x (2000).

12 Margerison, J. H. \& Corsellis, J. A. Epilepsy and the temporal lobes. A clinical, electroencephalographic and neuropathological study of the brain in epilepsy, with particular reference to the temporal lobes. Brain 89, 499-530, doi:10.1093/brain/89.3.499 (1966).

13 Seress, L. et al. Survival of mossy cells of the hippocampal dentate gyrus in humans with mesial temporal lobe epilepsy. J Neurosurg 111, 1237-1247, doi:10.3171/2008.11.JNS08779 (2009).

14 Swartz, B. E. et al. Hippocampal cell loss in posttraumatic human epilepsy. Epilepsia 47, 1373-1382, doi:10.1111/j.1528-1167.2006.00602.x (2006).

15 Buckmaster, P. S. \& Jongen-Relo, A. L. Highly specific neuron loss preserves lateral inhibitory circuits in the dentate gyrus of kainate-induced epileptic rats. J Neurosci 19, 9519-9529 (1999). 
16 Wong, R. K., Traub, R. D. \& Miles, R. Cellular basis of neuronal synchrony in epilepsy. Adv Neurol 44, 583-592 (1986).

17 Buckmaster, P. S., Wenzel, H. J., Kunkel, D. D. \& Schwartzkroin, P. A. Axon arbors and synaptic connections of hippocampal mossy cells in the rat in vivo. J Comp Neurol 366, 271-292 (1996).

18 Amaral, D. G., Scharfman, H. E. \& Lavenex, P. The dentate gyrus: fundamental neuroanatomical organization (dentate gyrus for dummies). Prog Brain Res 163, 3-22, doi:10.1016/S0079-6123(07)63001-5 (2007).

19 Henze, D. A., Wittner, L. \& Buzsaki, G. Single granule cells reliably discharge targets in the hippocampal CA3 network in vivo. Nat Neurosci 5, 790-795, doi:10.1038/nn887 (2002).

20 Pernia-Andrade, A. J. \& Jonas, P. Theta-gamma-modulated synaptic currents in hippocampal granule cells in vivo define a mechanism for network oscillations. Neuron 81, 140-152, doi:10.1016/j.neuron.2013.09.046 (2014).

21 Diamantaki, M., Frey, M., Berens, P., Preston-Ferrer, P. \& Burgalossi, A. Sparse activity of identified dentate granule cells during spatial exploration. Elife 5, doi:10.7554/eLife.20252 (2016).

22 Krook-Magnuson, E. et al. In vivo evaluation of the dentate gate theory in epilepsy. $J$ Physiol 593, 2379-2388, doi:10.1113/JP270056 (2015).

23 Hsu, D. The dentate gyrus as a filter or gate: a look back and a look ahead. Prog Brain Res 163, 601-613, doi:10.1016/S0079-6123(07)63032-5 (2007).

24 Hashimotodani, Y. et al. LTP at Hilar Mossy Cell-Dentate Granule Cell Synapses Modulates Dentate Gyrus Output by Increasing Excitation/Inhibition Balance. Neuron 95, 928-943 e923, doi:10.1016/j.neuron.2017.07.028 (2017).

25 Berthoux, C., Nasrallah, K. \& Castillo, P. E. BDNF-induced BDNF release mediates longterm potentiation. (in preparation) (2021).

26 Lin, T. W., Harward, S. C., Huang, Y. Z. \& McNamara, J. O. Targeting BDNF/TrkB pathways for preventing or suppressing epilepsy. Neuropharmacology 167, 107734, doi:10.1016/j.neuropharm.2019.107734 (2020).

27 McNamara, J. O. \& Scharfman, H. E. in Jasper's Basic Mechanisms of the Epilepsies (eds th et al.) (2012).

28 French, J. A. et al. Characteristics of medial temporal lobe epilepsy: I. Results of history and physical examination. Ann Neurol 34, 774-780, doi:10.1002/ana.410340604 (1993).

29 Lewis, D. V. et al. Hippocampal sclerosis after febrile status epilepticus: the FEBSTAT study. Ann Neurol 75, 178-185, doi:10.1002/ana.24081 (2014).

30 Shinnar, S. et al. Phenomenology of prolonged febrile seizures: results of the FEBSTAT study. Neurology 71, 170-176, doi:10.1212/01.wnl.0000310774.01185.97 (2008).

31 Raspall-Chaure, M., Chin, R. F., Neville, B. G. \& Scott, R. C. Outcome of paediatric convulsive status epilepticus: a systematic review. Lancet Neurol 5, 769-779, doi:10.1016/S1474-4422(06)70546-4 (2006).

32 Levesque, M. \& Avoli, M. The kainic acid model of temporal lobe epilepsy. Neurosci Biobehav Rev 37, 2887-2899, doi:10.1016/j.neubiorev.2013.10.011 (2013).

33 Rusina, E., Bernard, C. \& Williamson, A. The Kainic Acid Models of Temporal Lobe Epilepsy. eNeuro 8, doi:10.1523/ENEURO.0337-20.2021 (2021). 
34 Fredes, F. et al. Ventro-dorsal Hippocampal Pathway Gates Novelty-Induced Contextual Memory Formation. Curr Biol 31, 25-38 e25, doi:10.1016/j.cub.2020.09.074 (2021).

35 Zhang, X. et al. Stereotypical patterns of epileptiform calcium signal in hippocampal CA1, CA3, dentate gyrus and entorhinal cortex in freely moving mice. Sci Rep 9, 4518, doi:10.1038/s41598-019-41241-x (2019).

36 Kokaia, M. et al. Suppressed epileptogenesis in BDNF mutant mice. Exp Neurol 133, 215224, doi:10.1006/exnr.1995.1024 (1995).

37 He, X. P. et al. Conditional deletion of TrkB but not BDNF prevents epileptogenesis in the kindling model. Neuron 43, 31-42, doi:10.1016/j.neuron.2004.06.019 (2004).

38 Ramos, C. et al. Activation of extrasynaptic kainate receptors drives hilar mossy cell activity. https://www.biorxiv.org/content/10.1101/2021.05.01.442200v1 (2021).

39 Frerking, M., Malenka, R. C. \& Nicoll, R. A. Synaptic activation of kainate receptors on hippocampal interneurons. Nat Neurosci 1, 479-486, doi:10.1038/2194 (1998).

40 Castillo, P. E., Malenka, R. C. \& Nicoll, R. A. Kainate receptors mediate a slow postsynaptic current in hippocampal CA3 neurons. Nature 388, 182-186, doi:10.1038/40645 (1997).

41 Mulle, C. et al. Altered synaptic physiology and reduced susceptibility to kainate-induced seizures in GluR6-deficient mice. Nature 392, 601-605, doi:10.1038/33408 (1998).

42 Danielson, N. B. et al. In Vivo Imaging of Dentate Gyrus Mossy Cells in Behaving Mice. Neuron 93, 552-559 e554, doi:10.1016/j.neuron.2016.12.019 (2017).

43 GoodSmith, D. et al. Spatial Representations of Granule Cells and Mossy Cells of the Dentate Gyrus. Neuron 93, 677-690 e675, doi:10.1016/j.neuron.2016.12.026 (2017).

44 Senzai, Y. \& Buzsaki, G. Physiological Properties and Behavioral Correlates of Hippocampal Granule Cells and Mossy Cells. Neuron 93, 691-704 e695, doi:10.1016/j.neuron.2016.12.011 (2017).

45 Lauterborn, J. C. et al. Differential effects of protein synthesis inhibition on the activitydependent expression of BDNF transcripts: evidence for immediate-early gene responses from specific promoters. J Neurosci 16, 7428-7436 (1996).

46 Humpel, C. et al. Monitoring release of neurotrophic activity in the brains of awake rats. Science 269, 552-554, doi:10.1126/science.7624780 (1995).

47 Lowenstein, D. H., Seren, M. S. \& Longo, F. M. Prolonged increases in neurotrophic activity associated with kainate-induced hippocampal synaptic reorganization. Neuroscience 56, 597-604, doi:10.1016/0306-4522(93)90359-n (1993).

48 Binder, D. K., Croll, S. D., Gall, C. M. \& Scharfman, H. E. BDNF and epilepsy: too much of a good thing? Trends Neurosci 24, 47-53, doi:10.1016/s0166-2236(00)01682-9 (2001).

49 Binder, D. K., Routbort, M. J. \& McNamara, J. O. Immunohistochemical evidence of seizure-induced activation of trk receptors in the mossy fiber pathway of adult rat hippocampus. J Neurosci 19, 4616-4626 (1999).

50 Amaral, D. G. \& Witter, M. P. The three-dimensional organization of the hippocampal formation: a review of anatomical data. Neuroscience 31, 571-591, doi:10.1016/03064522(89)90424-7 (1989).

51 Katona, I. et al. Molecular composition of the endocannabinoid system at glutamatergic synapses. J Neurosci 26, 5628-5637, doi:10.1523/JNEUROSCI.0309-06.2006 (2006). 
52 Jensen, K. R., Berthoux, C., Nasrallah, K. \& Castillo, P. E. Multiple cannabinoid signaling cascades powerfully suppress recurrent excitation in the hippocampus. Proc Natl Acad Sci U S A 118, doi:10.1073/pnas.2017590118 (2021).

53 Marsicano, G. et al. CB1 cannabinoid receptors and on-demand defense against excitotoxicity. Science 302, 84-88, doi:10.1126/science.1088208 (2003).

54 Monory, K. et al. The endocannabinoid system controls key epileptogenic circuits in the hippocampus. Neuron 51, 455-466, doi:10.1016/j.neuron.2006.07.006 (2006).

55 Katona, I. Cannabis and Endocannabinoid Signaling in Epilepsy. Handb Exp Pharmacol 231, 285-316, doi:10.1007/978-3-319-20825-1_10 (2015).

56 Scharfman, H. E., Goodman, J. H., Sollas, A. L. \& Croll, S. D. Spontaneous limbic seizures after intrahippocampal infusion of brain-derived neurotrophic factor. Exp Neurol 174, 201-214, doi:10.1006/exnr.2002.7869 (2002).

57 He, X. P., Pan, E., Sciarretta, C., Minichiello, L. \& McNamara, J. O. Disruption of TrkBmediated phospholipase Cgamma signaling inhibits limbic epileptogenesis. J Neurosci 30, 6188-6196, doi:10.1523/JNEUROSCI.5821-09.2010 (2010).

58 Heinrich, C. et al. Increase in BDNF-mediated TrkB signaling promotes epileptogenesis in a mouse model of mesial temporal lobe epilepsy. Neurobiol Dis 42, 35-47, doi:10.1016/j.nbd.2011.01.001 (2011).

59 Liu, G. et al. Transient inhibition of TrkB kinase after status epilepticus prevents development of temporal lobe epilepsy. Neuron 79, 31-38, doi:10.1016/j.neuron.2013.04.027 (2013).

60 Croll, S. D. et al. Brain-derived neurotrophic factor transgenic mice exhibit passive avoidance deficits, increased seizure severity and in vitro hyperexcitability in the hippocampus and entorhinal cortex. Neuroscience 93, 1491-1506, doi:10.1016/s03064522(99)00296-1 (1999).

61 Falcicchia, C. et al. Seizure-Suppressant and Neuroprotective Effects of Encapsulated BDNF-Producing Cells in a Rat Model of Temporal Lobe Epilepsy. Mol Ther Methods Clin Dev 9, 211-224, doi:10.1016/j.omtm.2018.03.001 (2018).

62 Schmidt-Hieber, C., Jonas, P. \& Bischofberger, J. Enhanced synaptic plasticity in newly generated granule cells of the adult hippocampus. Nature 429, 184-187, doi:10.1038/nature02553 (2004).

63 Larimer, P. \& Strowbridge, B. W. Nonrandom local circuits in the dentate gyrus. J Neurosci 28, 12212-12223, doi:10.1523/JNEUROSCI.3612-08.2008 (2008).

64 Lysetskiy, M., Foldy, C. \& Soltesz, I. Long- and short-term plasticity at mossy fiber synapses on mossy cells in the rat dentate gyrus. Hippocampus 15, 691-696, doi:10.1002/hipo.20096 (2005).

65 Hedrick, T. P. et al. Excitatory Synaptic Input to Hilar Mossy Cells under Basal and Hyperexcitable Conditions. eNeuro 4, doi:10.1523/ENEURO.0364-17.2017 (2017).

66 Manvich, D. F. et al. The DREADD agonist clozapine N-oxide (CNO) is reversemetabolized to clozapine and produces clozapine-like interoceptive stimulus effects in rats and mice. Sci Rep 8, 3840, doi:10.1038/s41598-018-22116-z (2018).

67 Whissell, P. D., Tohyama, S. \& Martin, L. J. The Use of DREADDs to Deconstruct Behavior. Front Genet 7, 70, doi:10.3389/fgene.2016.00070 (2016). 
68 Racine, R. J. Modification of seizure activity by electrical stimulation. II. Motor seizure. Electroencephalogr Clin Neurophysiol 32, 281-294 (1972).

69 Mizrahi, A., Crowley, J. C., Shtoyerman, E. \& Katz, L. C. High-resolution in vivo imaging of hippocampal dendrites and spines. J Neurosci 24, 3147-3151, doi:10.1523/JNEUROSCI.5218-03.2004 (2004).

70 Pachitariu, M., Stringer, C. \& Harris, K. D. Robustness of Spike Deconvolution for Neuronal Calcium Imaging. J Neurosci 38, 7976-7985, doi:10.1523/JNEUROSCI.333917.2018 (2018). 

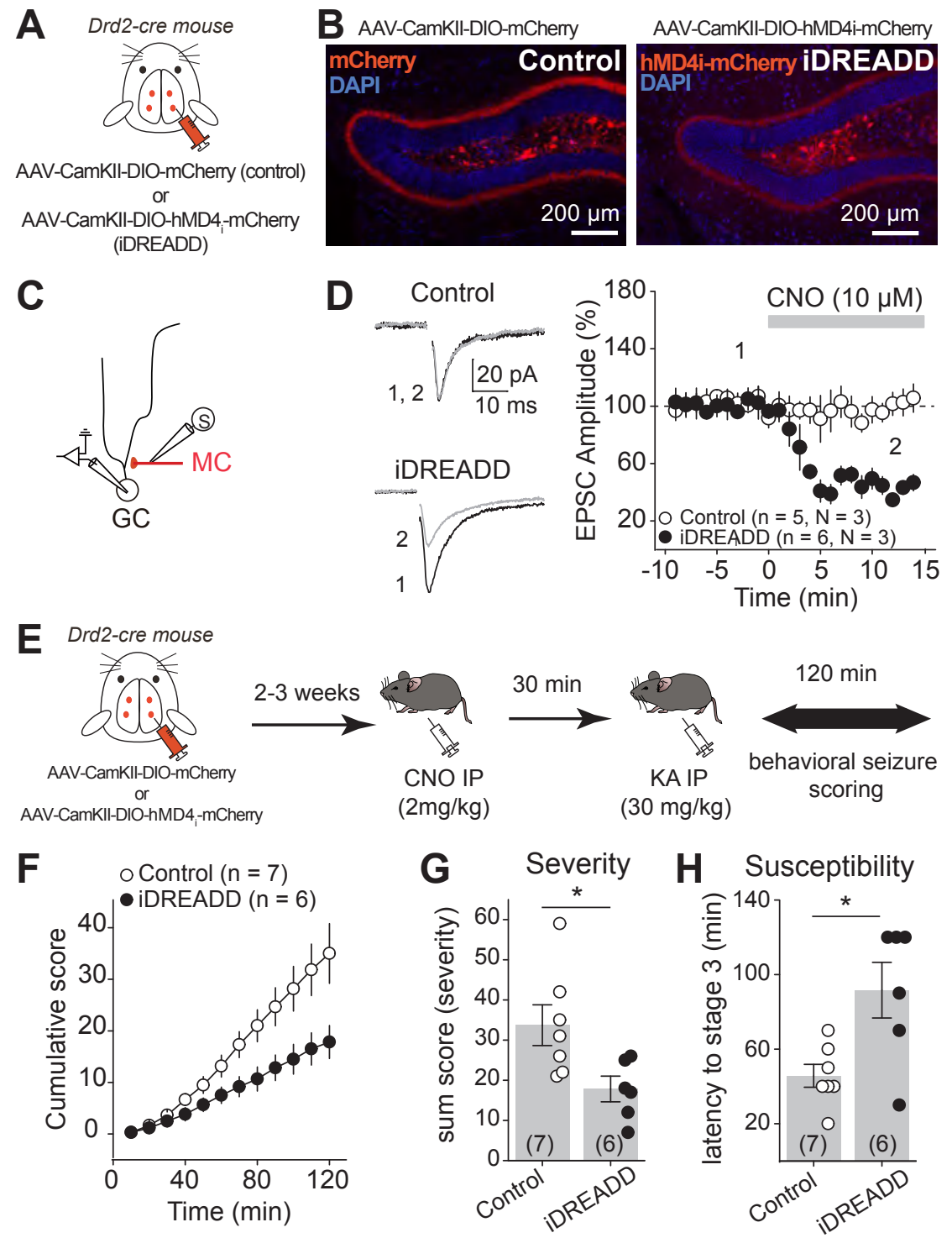

Figure 1: Chemogenetic silencing of MCs reduced acute kainic acid-induced seizures 
A head-fixed 2 photon

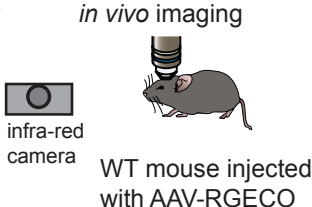

B

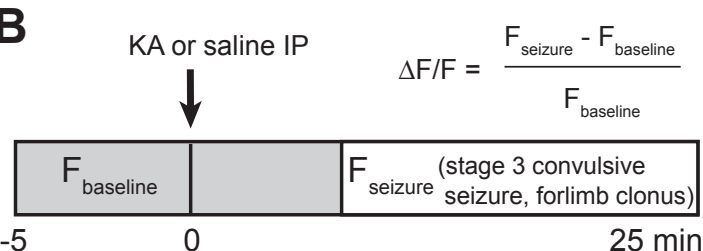

C before KA

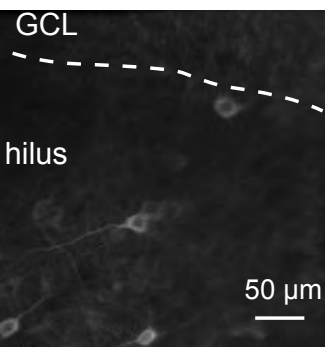

after KA

D $\quad \mathrm{MC}$

GC
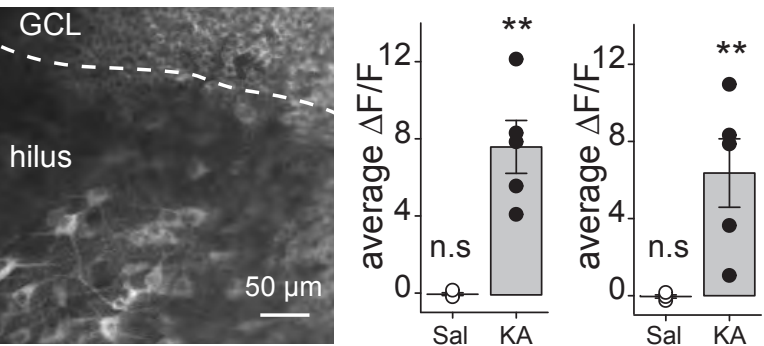

E
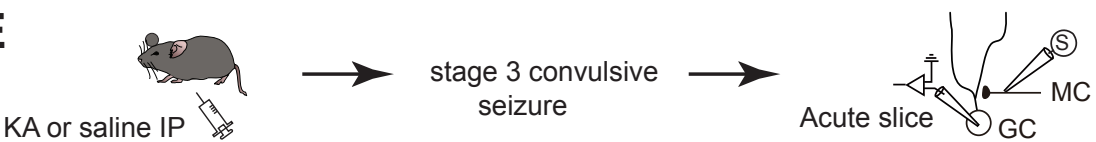

$\mathbf{F}$
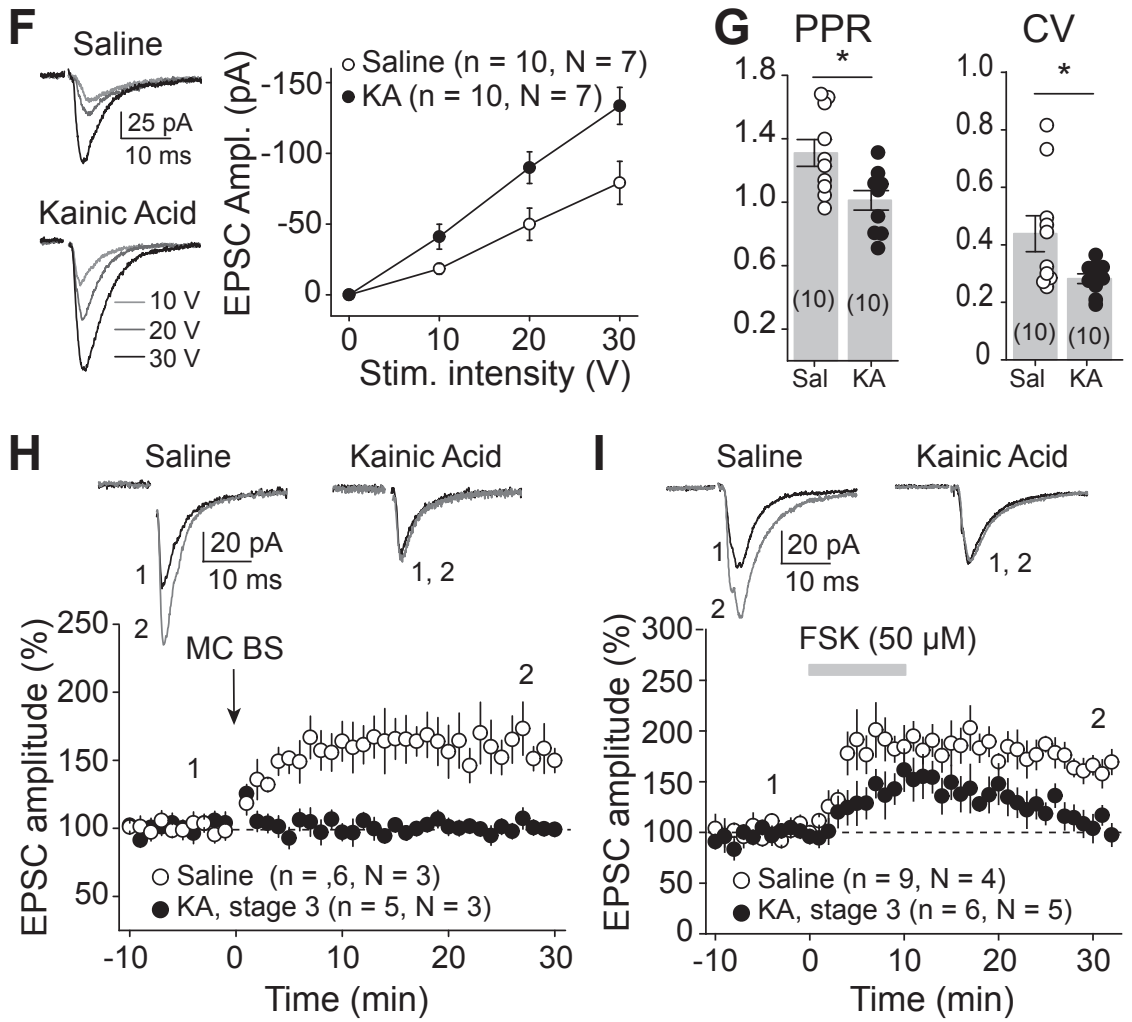

Figure 2: Initial convulsive seizures increased DG excitatory neuron activity and MC-GC synaptic strength 
A BDNFflfl mouse

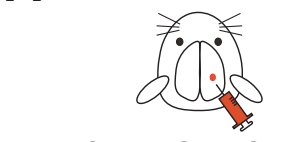

AAV-CamKII-Cre-mCherry (KO)

or

AAV-CamKII-mCherry (control)

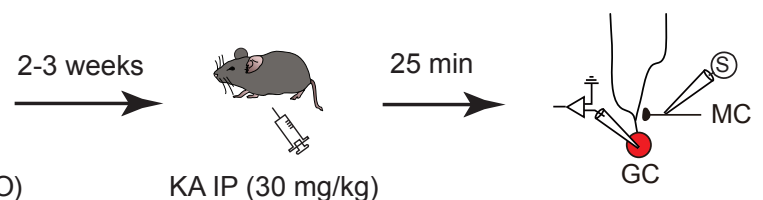

or

Saline IP
Acute slice preparation
B

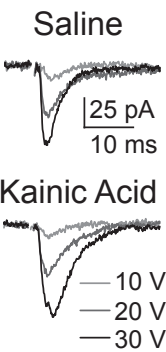

D

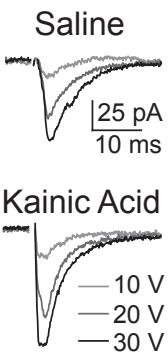

BDNF cKO

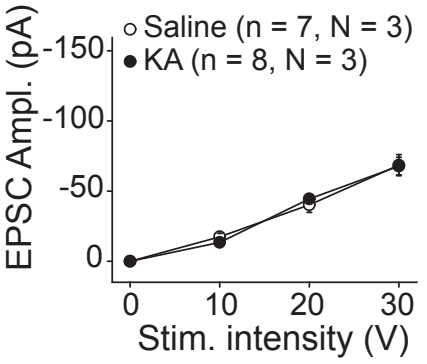

Control

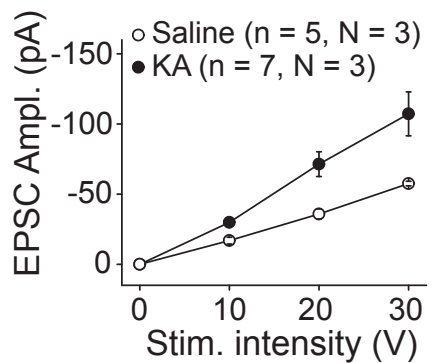

C BDNF CKO

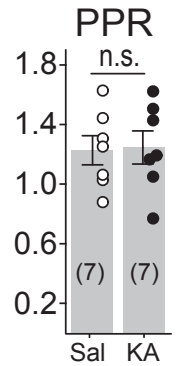

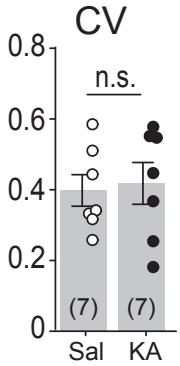

E

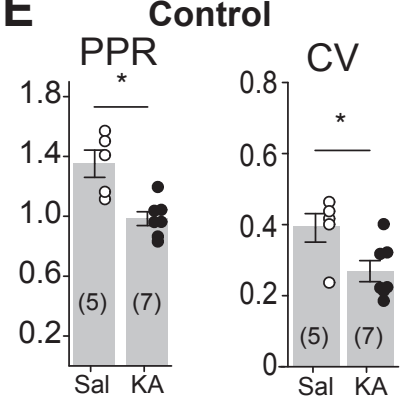

$\mathbf{F}$

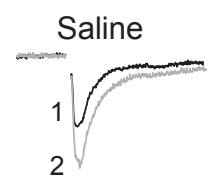

BDNF cKO
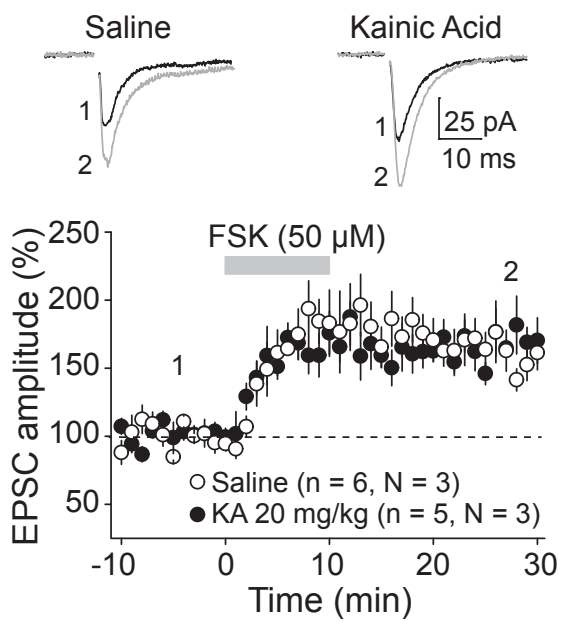

G

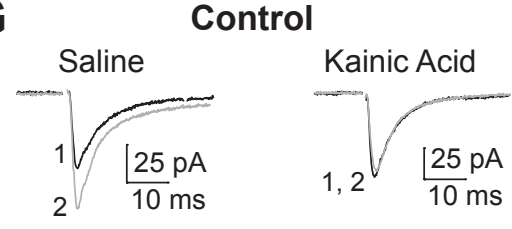

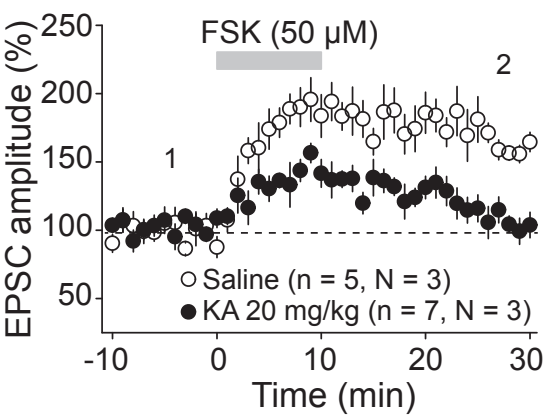

Figure 3: Seizure-induced MC-GC synaptic strengthening required postsynaptic BDNF 
A BDNF"nn mouse
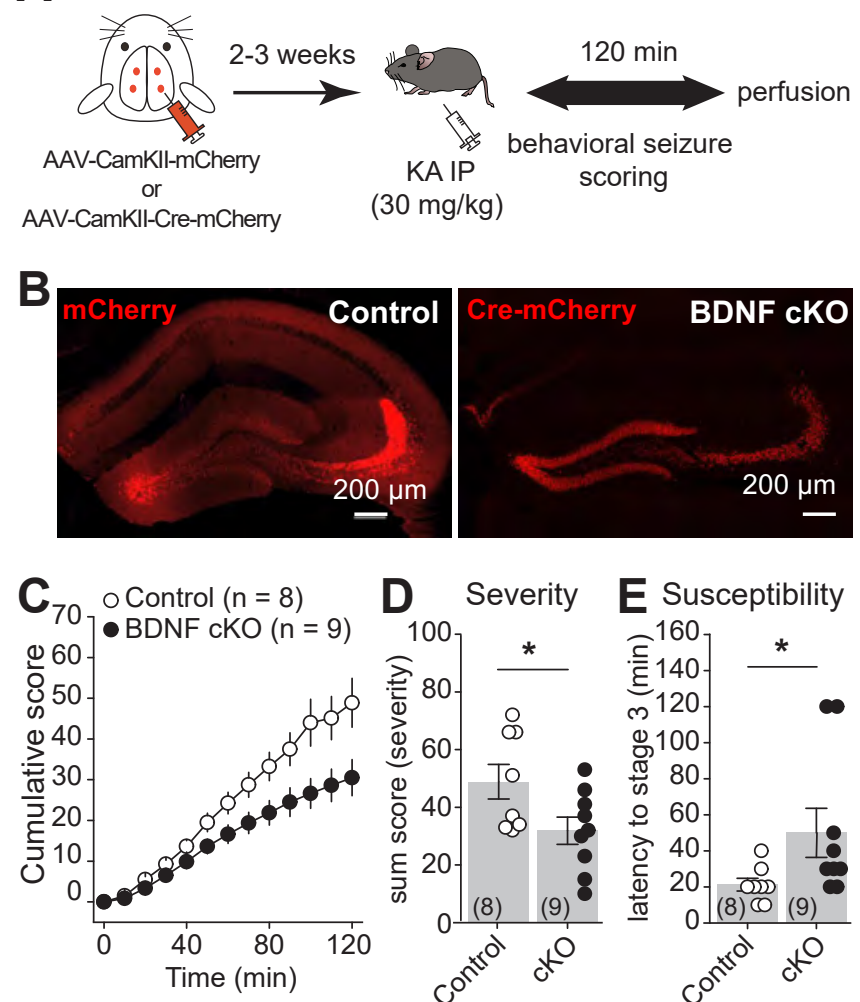

D Severity E Susceptibility
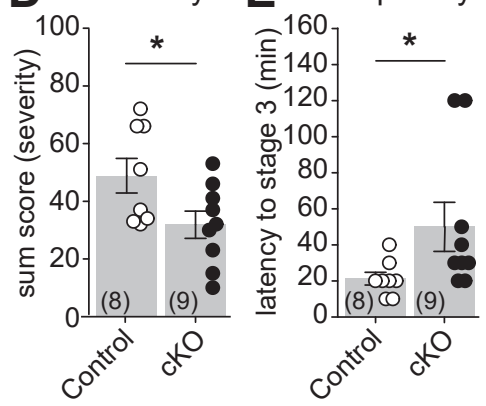

Figure 4: Knocking out BDNF from hippocampal excitatory neurons reduced KA-induced seizures 
A Drd2-Cre mouse
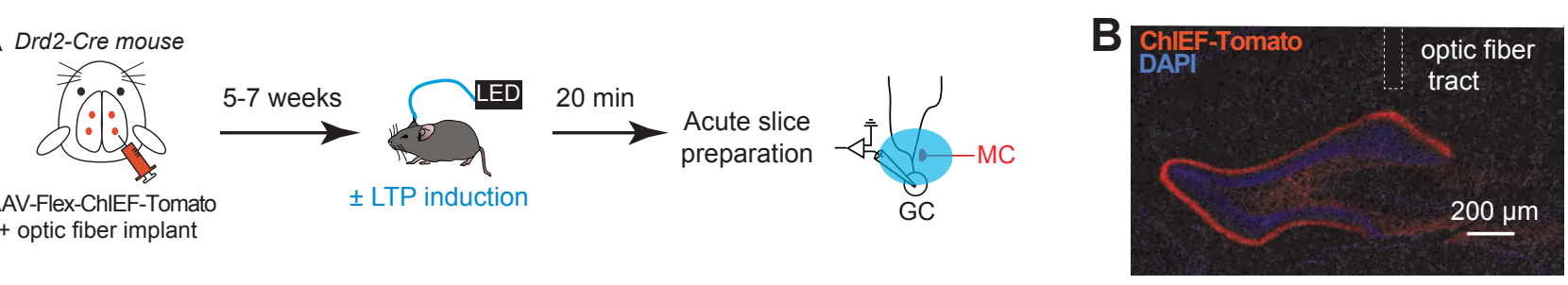

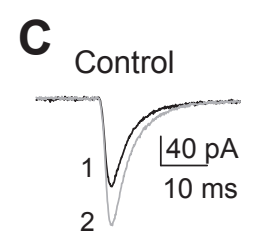

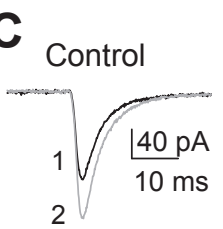

after in vivo LTP

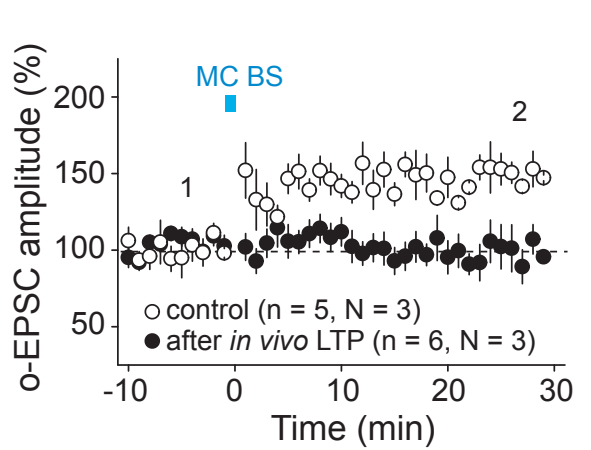

D
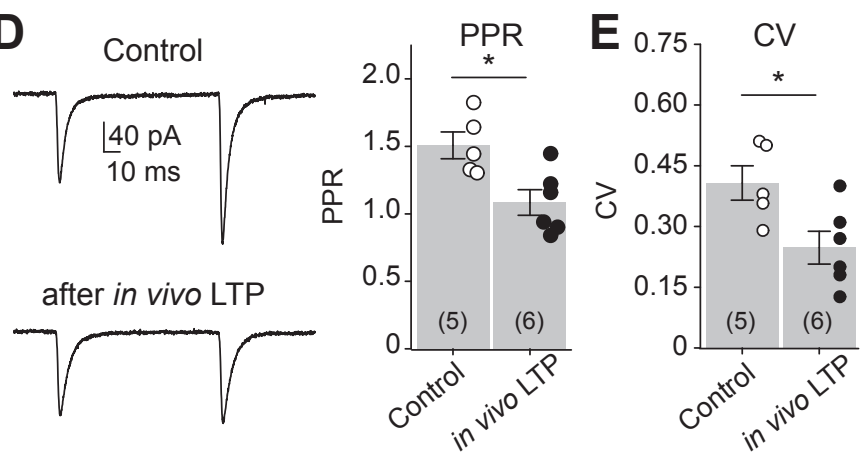

$\mathbf{F}$

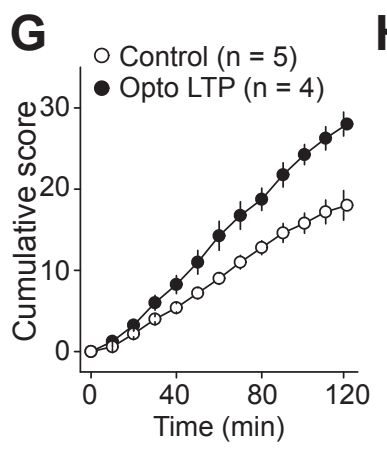

H Severity I Susceptibility
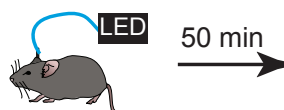

seizure scoring

\pm LTP induction

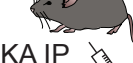

$120 \mathrm{~min}$

$(20 \mathrm{mg} / \mathrm{kg})$

perfusion

120 min
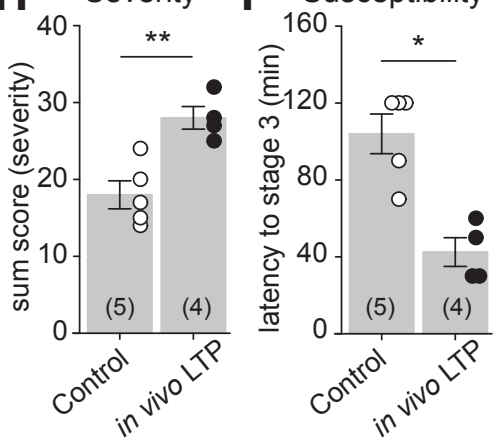

Figure 5: In vivo induction of MC-GC LTP promoted seizures 
A

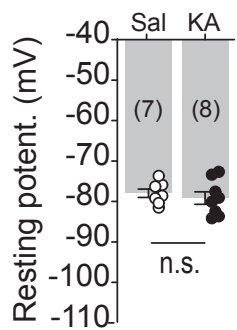

B

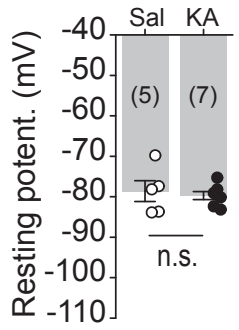

BDNF cKO
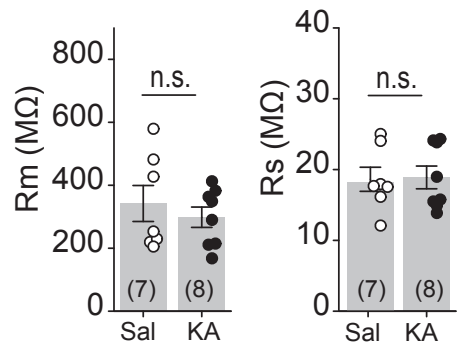

\section{Control}

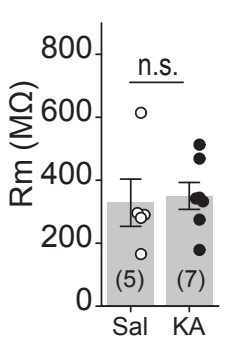

Figure S1 (related to Figure 3): BDNF cKO did not alter GC membrane properties

(A) $B d n f^{f / f l}$ mice were injected with Cre-expressing virus (AAV-CaMKII-Cre-mCherry, BDNF cKO). Whole cell recordings were performed in Cre-mCherry-expressing GCs. Resting potential (saline: $-77.3 \pm 1.0 \mathrm{mV}, \mathrm{n}=7$; KA: $-79.2 \pm 1.6$ $\mathrm{mV}, \mathrm{n}=8$; saline vs $\mathrm{KA}: \mathrm{p}>0.05$, unpaired t-test), membrane resistance $(\mathrm{Rm}$, saline: $340.9 \pm 57.0 \mathrm{M} \Omega, \mathrm{n}=7 ; \mathrm{KA}: 298.5 \pm$ $32.9 \mathrm{M} \Omega, \mathrm{n}=8$; saline vs $\mathrm{KA}: \mathrm{p}>0.05$, unpaired t-test) and series resistance (Rs, saline: $18.3 \pm 1.7 \mathrm{M} \Omega, \mathrm{n}=7 ; \mathrm{KA}$ : $18.9 \pm$ 1.6 $\mathrm{M} \Omega, n=8$; saline vs $K A: p>0.05$, unpaired t-test) were not different in $K A$ vs saline-treated.

(B) $B d n f^{\prime / f l}$ mice were injected with a control virus (AAVCaMKII-mCherry). Membrane properties were similar in KAand saline-injected mice (Resting potential, saline: -78.6 \pm 2.6 $\mathrm{mV}, \mathrm{n}=5$; KA: $-79.7 \pm 1.0 \mathrm{mV}, \mathrm{n}=7$; saline vs $\mathrm{KA}: \mathrm{p}>0.05$, unpaired t-test; $R m$, saline: $328.7 \pm 75.2 \mathrm{M} \Omega, \mathrm{n}=5$; KA: 350.2 $\pm 42.5 \mathrm{M} \Omega, n=7$; saline vs $\mathrm{KA}: \mathrm{p}>0.05$, Mann Whitney test; Rs, saline: $19.7 \pm 2.1 \mathrm{M} \Omega, \mathrm{n}=5$; $\mathrm{KA}: 19.6 \pm 1.8 \mathrm{M} \Omega, \mathrm{n}=7$; saline vs KA: $p>0.05$, unpaired t-test). 

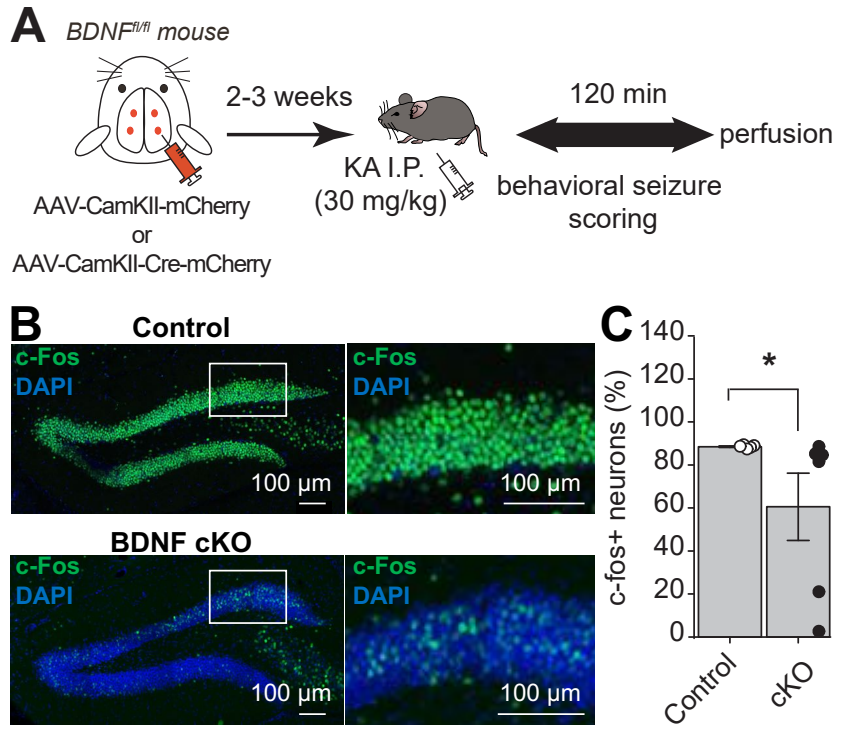

Figure S2 (related to Figure 4): Neuronal BDNF cKO protected DG gating properties

(A) Diagram of the experimental timeline. (B and C) Confocal images (B) and quantification (C) showing how BDNF KO reduced the number in c-fos+ neurons in the DG of KA-injected mice (control: $88.5 \pm 0.3 \%$ of c-Fos + neurons, $N=5$ mice; BDNF cKO: $60.5 \pm 15.6 \%$ of c-Fos + neurons, $\mathrm{N}=5$ mice; control vs BDNF cKO: $p<0.05$, Mann Whitney test). 


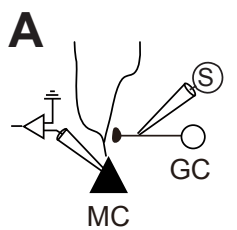

C

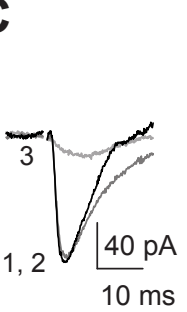

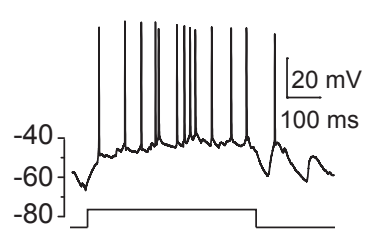

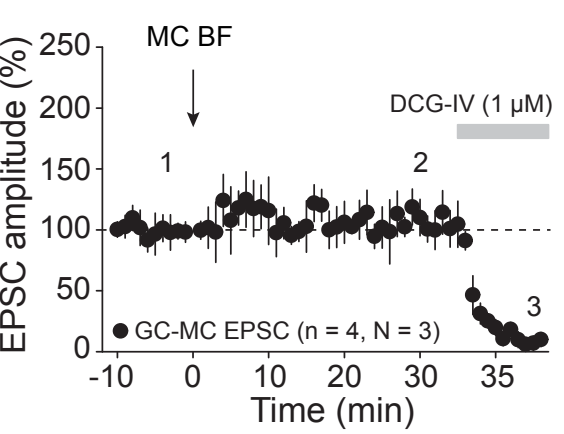

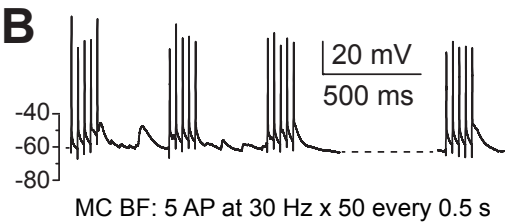

D Membrane Resist.

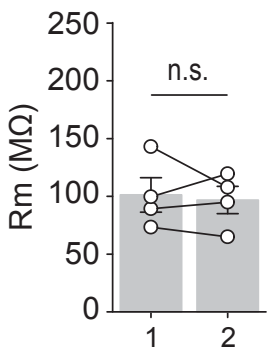

Figure S3 (related to Figure 5): MC Burst Firing did not induce long-term changes at GC-MC synapse

(A) Left, diagram showing the recording conditions. Whole cell recordings were performed in MCs in response to GC axon stimulation, while both excitatiory and inhibitory transmission were left intact. Right, voltage response elicited by a depolarizing current step injection (shown below) illustrating typical MC firing pattern.

(B) Current clamp recordings showing the induction protocol. MC Burst Firing (MC BF) was composed of $5 \mathrm{AP}$ at $30 \mathrm{~Hz}$, repeated 50 times, every $0.5 \mathrm{~s}$.

(C) Representative traces and time course summary plot showing that application of MC BF did not induce any significant long-term change of GC-MC EPSC amplitude (105.9 $\pm 10.7 \%$ of baseline, $n=4, p>0.05$, paired t-test ).

DCG-IV was applied at the end of each experiment to confirm GC input nature. (D) MC membrane resistance was not affected by MC BF application (before MC BS: $101.3 \pm 14.9 \mathrm{M} \Omega$, after MC BS: $96.8 \pm 11.8 \mathrm{M} \Omega$, before vs after MC BS: $p>0.05$, paired t-test, $n=4$ ). 